\title{
Quantification, characterization and description of synergy and antagonism in the antioxidant response.
}

Prieto M.A. , Murado M.A., \& Vázquez J.A.

Grupo de Reciclado e Valorización de Materiais Residuais (REVAL), Instituto de Investigacións Mariñas (IIM), Consejo Superior de Investigaciones Científicas (CSIC), r/Eduardo Cabello, 6. Vigo36208, Galicia, Spain.

*Corresponding author

E-mail: michaelumangelum@gmial.com

Tel.: +34986214469; +34986231930

Fax: +34986292762

\begin{abstract}
This paper illustrates a methodological procedure to determine the synergistic and antagonistic effects of combining antioxidant agents. Current methods to determinate the interactive actions of antioxidants have been rejected, and we attempt to address this issue by incorporating well-established ideas from different existing fields. Two mathematical models are proposed, which provide explicit algebraic forms and generalize the classical hypothesis of independent action and concentration addition as they are defined in the dose-response relationships. In addition, a comprehensive index to summarize all the complex responses in one single value is proposed, which allows the extraction and identification of the relevant aspects. Although the approach could be directly expanded to other types of classical antioxidant methods, two complex scenarios were recreated using different but complementary well-known kinetic antioxidant methods, which are fairly representative of lipidic and hydrophilic oxidation processes. Meanings of synergy and antagonism concepts were found that describe and characterize the interactions between several pairs of commercial antioxidants in a statistically consistent form. The results also provided some evidence of a more basic character, which, if transferable to more realistic food matrices in the food industry, may guide the development and evaluation of food products and processes, as well as the study of mechanisms underlying different phenomena that may affect the quality of products.
\end{abstract}

Keywords: dose-response analysis; synergy and antagonism; mechanisms of interaction; antioxidant interaction; $\beta$-carotene and crocin bleaching assay

\section{INTRODUCTION}

An important and characteristic problem of any system (as defined in the Bertalanffy's theory: a set of interacting elements) is to determinate whether the joint effect of two or more elements on the system behavior is directly deducible from the individual effects of the elements. This issue has a long history of controversy whose first known attempt to solve it dates back to Aristotle, and it is frequently stated by replacing the expression "directly deducible from" with "the sum of", which significantly change the focus. Thus, in the field of antioxidant action, the concepts of synergy and antagonism are often characterized as those interactions of two (or more) antioxidants that are greater (synergy) or lesser (antagonism) than the sum of the individual effects (Jia et al., 1998; Marinova et al., 2008; Parker et al., 2010; Yang et al., 2009). Such a characterization is not acceptable for two reasons.

First, it postulates that the joint effect in the absence of interactions is the sum of the individual effects, which is an especially simplistic case and not applicable to asymptotic responses, such as those involved in the action of anti- and pro-oxidant agents. Indeed, the sum of two individual responses is meaningless if it exceeds the asymptotic response of the system. In fact, the referent of any phenomenon that perturbs the joint action of two agents is that joint action in the absence of 
perturbation (not the individual actions), a situation that is often called as the "null interaction". Consequently, the first condition to decide the possible presence of synergistic or antagonistic effects is to define the null interaction. A second difficulty arises from the common tools applied to characterize the antioxidant action. Despite abundant criticism (Labuza \& Dugan, 1971; Murado \& Vázquez, 2010; Prieto et al., 2012; 2012a; Terpinc \& Abramovič, 2010; Özilgen \& Özilgen, 1990), such a characterization frequently disregards the kinetic aspects of the oxidation process and its inhibition. Although this objection has a less theoretical significance than the first one, its practical consequence is that the results may be poorly suited to discern the joint effect of two antioxidants.

This paper pursues a solution for each of these objections by using concentration-time response models applied to the $\beta$-carotene ( $\beta C M)$ (Marco, 1968; Miller, 1971) and crocin bleaching (CM) (Bors et al. 1984) methods - extensively used to quantify antioxidant and prooxidant activities- to assess the synergistic or antagonistic interactions between several pairs of well-known antioxidants. Their respective protocols have been repeatedly revised and improved, and they are optimized at present (Prieto et al., 2012; 2012a). They are appropriate for lipophilic and hydrophilic matrices and can provide useful complementary information in the study of complex natural extracts containing components with a variable degree of polarity (Prieto et al., 2013). $\beta$-carotene is a lipophilic oxidizable substrate that can join the system of lipidic micelles in which the oxidation reaction is accomplished. The method is especially sensitive to oxidation modifying agents in a lipidic environment, and it produces a very low response with hydrophilic antioxidants, even powerful ones (polar paradox). Complementarily, crocin is a hydrophilic oxidizable substrate, and lipophilic oxidation modifiers, even powerful ones, produce very low responses in the aqueous system that characterize the application of this method (apolar paradox). These assays were selected because they provide an optimized response system that is fairly representative of the lipidic and hydrophilic oxidation processes, especially accurate, reproducible and yields a low experimental error.

The first problem, which consists of distinguishing between null interaction and synergistic or antagonistic effects was studied by generalizing the classical approaches (Berenbaum, 1985a; 1985b; Bliss, 1937; 1939; Loewe \& Muischnek, 1926; Greco et al., 1995) applied in the dose-response area (not free either of debate about the interactive effects) and others (Qin et al.,2011; Hewlett \& Plackett, 1964; Gessner, 1988; Rovati \& Nicosia, 1994; Baldwin \& Roling, 2009). The second difficulty was solved by defining the response of the system to the simultaneous action of two antioxidants through a single value obtained from a kinetic description as previously discussed (Dávalos et al., 2004; Huang et al., 2008; Naguib, 2000; Prieto et al., 2012).

The proposed generalized procedures for the joint action of several well-known antioxidants produced consistent results in all cases. In addition, it provided some evidence of a more basic character, which could be transferable to the general field of the in vivo dose-response relationships.

\section{MATERIAL AND METHODS}

\subsection{Methods to assess the antioxidant activity}

\subsubsection{Equipment and reagents}

- Equipment: Multiskan spectrum microplate photometer using polypropylene plates with 96 wells.

- Antioxidants: butyl-hydroxyanisole (BHA); ; propyl 3,4,5-trihydroxybenzoate (Propyl gallate; PG); butyl-hydroxytoluene (BHT); 6-ethoxy-2,2,4-trimethyl-1,2-dihydroquinoline (Ethoxyquin; ETO); 6-hydroxy-2,5,7,8-tetramethylchroman-2-carboxylic acid (Trolox; TRO); and (2R)-2,5,7,8tetramethyl-2-[(4R,8R)-(4,8,12-trimethyltridecyl)]-6-chromanol ( $\alpha$-tocopherol; TOC); manganese sulfate (Mn); (5R)-[(1S)-1,2-dihydroxyethyl]-3,4-dihydroxyfuran-2(5H)-one (Ascorbic acid; AA).

- Crocin bleaching reagent: $4 \mathrm{mg}$ of Crocin and $75 \mathrm{mg}$ of AAPH were dissolved in 25 and $5 \mathrm{~mL}$, respectively, of $100 \mathrm{mM}$ Briton buffer, $\mathrm{pH}=5.5$, in Mili-Q water at $40^{\circ} \mathrm{C}$. Both solutions must be prepared and mixed just before use. 
- $\beta$-Carotene bleaching reagent: $4 \mathrm{mg}$ of $\beta$-Carotene, $0.5 \mathrm{ml}$ of linoleic acid and $4 \mathrm{~g}$ of Tween-40 were dissolved in $20 \mathrm{ml}$ of chloroform and the chloroform was evaporated in a rotary evaporator $\left(40^{\circ} \mathrm{C} / \sim 15 \mathrm{~min}\right)$. One mililiter of the oily residue is added to $30 \mathrm{ml}$ of Mili-Q water at $\mathrm{pH}=6.5$ (Briton buffer $100 \mathrm{mM}$ ) at the assay temperature $\left(45^{\circ} \mathrm{C}\right)$.

\subsubsection{Procedure}

Microplate assays were carried out based on a complete design (more details are provided in the appendix and Figure A1) that consisted of $8 \times 8$ arrays of two antioxidant mixtures at equally increasing concentrations, which were freshly prepared in water:ethanol (9:1). Thus, $25 \mu$ l of each antioxidant solution was added to each well containing $250 \mu \mathrm{l}$ of the preheated reagent $\left(\mathrm{CM}: 37^{\circ} \mathrm{C}\right.$ and $\beta \mathrm{CM}$ : $\left.45^{\circ} \mathrm{C}\right)$. The apparatus was programmed for $200 \mathrm{~min}$ at $37^{\circ} \mathrm{C} / 450 \mathrm{~nm}(\mathrm{CM})$ and $45^{\circ} \mathrm{C} / 470 \mathrm{~nm}(\beta \mathrm{CM})$, with agitation at 660 cycles/min (1 mm amplitude), which was only interrupted for readings at $3 \mathrm{~min}$ intervals (covering initiation, propagation and asymptotic phases with a total of 64 independent kinetic measures per each of the 64 concentration combinations). By using the antioxidants listed in the materials section, 21 combinations were performed for each method, including those in which the pair of antioxidant is the same antioxidant (used simply as a control). The concentration range applied is presented in Table 3.

\subsubsection{Selection of a single value to assess the response}

The usual methods were based on comparing the oxidation rate, half-life, lag phase and the area under the curve $(A U C)$ of a given substrate in the presence of increasing concentrations of the studied antioxidant. All antioxidant combinations were first analyzed by comparing the four above mentioned parameters. Although the results showed that all of them lead to similar conclusions, the use of the area under the curve $(A U C)$ proved to be a highly robust criterion, which summarizes in a single and direct datum the global feature of any kinetic profile, while avoiding some minor drawbacks (affecting mainly the smoothness of the values) that emerge when other parameters are used.

This criterion is frequently applied for a dose-time response of an antioxidant standardizing the responses in relation to $A U C$ obtained for the control, which leads to the formulation of the relative area units $(R A U)$, as defined by other authors (Dávalos et al., 2004; Huang et al. 2002; Naguib, 2000). To obtain the $R A U$ values, the response first needs to be compute in terms of area units, which can be calculated by any numerical integration method. For example, if $R_{t}$ is the response to a set of $t$ times, the area units under the curve can be calculated using numerical methods of integration, such as the trapezoidal rule:

$$
A U C=\frac{1}{2} h_{t}\left[R_{t=0}+R_{t=n}\right]+h_{t} \sum_{t=1}^{n-1} R_{t}
$$

in which $h_{\mathrm{t}}$ is the kinetic interval used $\left(3 \mathrm{~min}\right.$ ). Consequently, if $A U C_{0}$ and $A U C_{c}$ are the area units corresponding to the kinetic profiles found in the absence and presence of an antioxidant concentration $c$, respectively, the $R A U$ value that increases with the concentration and the power of the antioxidant can be defined as follows:

$$
R A U(A)=A U C_{0}-A U C_{c}
$$

The $A U C_{0}$ is also the maximum response achievable $\left(R U A_{\max }\right)$. Consequently values of $R A U$ obtained can be standardized in responses $(R)$ over a scale [0,1], which facilitates comparisons:

$R=R A U / R A U_{\max }$ 
The variation of $R A U$ as function of any agent can be described satisfactorily using the Weibull cumulative distribution function (Weibull, 1951), thus the effect of increasing concentrations $\left(A_{i}\right)$ of an antioxidant $(A)$ can be described in general terms as follows:

$$
R(A)=K\left\{1-\exp \left[-\ln 2(A / m)^{a}\right]\right\} \quad \text { briefly; } \quad R=W(A ; K, m, a)
$$

where $K$ is the asymptotic response, $m$ is the concentration producing the half-maximal response and $a$ is a shape parameter related to the slope. This equation is very versatile: if $a<1$, it fits the potential profiles produced by the model of (Terpinc \& Abramovič, 2010), if $a=1$, it describes a first order kinetic model, and if $a>1$, it produces a variety of sigmoidal profiles that are the common solution for the system.

\subsection{Dose-response theory}

\subsubsection{The null interaction forms}

Two basic types of null interaction are conventional considered in the dose-response field. For two agents, these interactions are defined as follows:

\section{(a) Independent action}

This mode of action assumes that the agents act via different mechanisms, each of which reaches an asymptotic maximum as a result of a statistically independent phenomenon. Under this premise, probability theory defines the response, as the sum of the probabilities of the individual phenomena minus the probability of their joint occurrence (Bliss, 1939). Consequently, if $R_{\mathrm{c}}$ is the response to the joint action of the concentrations $c_{1}$ and $c_{2}$, and $R_{\mathrm{c} 1}$ and $R_{\mathrm{c} 2}$ the individual responses at the same concentrations, the total response can be established:

$$
R_{c}=R_{c 1}+R_{c 2}\left(1-R_{c 1}\right)
$$

An expression that is easily generalized to more than two agents can be obtained by writing $R_{c 1}$ as $1-$ $\left(1-R_{c 1}\right)$ and substituting it in (5):

$$
R_{c}=1-\left(1-R_{c 1}\right)+R_{c 2}\left(1-R_{c 1}\right)=1-\left(1-R_{c 1}\right)\left(1-R_{c 2}\right)
$$

\section{(b) Concentration addition}

The typical formulation (Berenbaum, 1985a; 1985b) does not define the null interaction as a relationship between individual responses, but uses the following criteria: the concentration $(c)$ of an agent whose action obeys the equation $R=f(c)$ can be considered a fictitious combination of the concentrations $c_{1}$ and $c_{2}\left(c=c_{1}+c_{2}\right)$. Under these conditions, the response to $c$ will be given by the equation $R=f(c)$ with $c=c_{1}+c_{2}$. If the response to a mixed dose of two agents behaves as the response to the "mixed" dose of the same agent, it is accepted that the interaction between them is null. This fact indicates that any agent concentration can be effectively substituted by the equivalent concentration of the other one.

\subsubsection{The combined action of two antioxidants with and without interactions}

The simultaneous action of two antioxidants can occur according to any of both modes of action listed above, even in very simple processes (see Figure 1). Therefore, to propose explicit algebraic forms for these modes of action in the case of the action of antioxidant agents requires applying the equation (4) in the framework of the $I A$ and $C A$ hypotheses as follows: 
The basic model (null interaction) is directly obtained by transferring the equation (4) into (6):

$$
R=1-\left[1-W\left(A_{1} ; K_{1}, m_{1}, a_{1}\right)\right]\left[1-W\left(A_{2} ; K_{2}, m_{2}, a_{2}\right)\right]
$$

Any interaction necessarily implies that the presence of an antioxidant alters the parameters of the response to the other in an effect that can be unidirectional or reciprocal. We have previously proven (Murado et al., 2011) that to change the response of Weibull's equation as function of the effect of another variable can be achieved by multiplying $K$ and $m$ parameters by a hyperbolic perturbation term that includes the variable responsible for the alteration. Because the variable that perturbs the response to an antioxidant $A_{i}$ is another antioxidant $A_{j}$, the perturbation terms will have the following form:

$$
v_{\theta i}=\left(1+b_{\theta i} A_{j}\right) /\left(1+c_{\theta i} A_{j}\right) ;(\mathrm{i} \neq \mathrm{j})
$$

where $v_{\theta \mathrm{i}}$ is the factor that multiplies the $\theta$ parameter $(K$ or $m)$ of the response to $A_{\mathrm{i}}$, and it is a function of the concentration of the antioxidant $A_{\mathrm{j}}$ with fitting coefficients $b_{\theta \mathrm{i}}$ and $c_{\theta \mathrm{i}}$. In the most complex scenario, assuming reciprocal perturbations in both parameters, the model (7) turns into the following:

$$
R=1-\left[1-W\left(A_{1} ; K_{1} v_{k 1}, m_{1} v_{m 1}, a_{1}\right)\right]\left[1-W\left(A_{2} ; K_{2} v_{k 2}, m_{2} v_{m 2}, a_{2}\right)\right]
$$

Expression (5) clarifies the meaning of an additional possible modification. Indeed, when the individual responses are denoted as $W_{\mathrm{i}}$, it becomes evident that the $W_{1} W_{2}$ term of the joint response $\left(R=W_{1}+W_{2}-W_{1} W_{2}\right)$ is associated with the hypothesis of statistical independence. Therefore, this term will be modified if this independence is altered by any global cooperative or competitive effect. Thus, a generalized $I A$ model, in its most complex form, can be written as follows:

$$
R=W\left(D_{1} ; K_{1} v_{k 1}, m_{1} v_{m 1}, a_{1}\right)+W\left(D_{2} ; K_{2} v_{k 2}, m_{2} v_{m 2}, a_{2}\right)\left[1-s \times W\left(D_{1} ; K_{1} v_{k 1}, m_{1} v_{m 1}, a_{1}\right)\right]
$$

where the value of the coefficient $s$ becomes greater or lesser than 1 depending on the predominance of competitive or cooperative effects, respectively. It should be noted that even though model (10) includes all the possible theoretical interactions, much simpler situations are normally found (because several $\left.v_{\theta \mathrm{i}}=1\right)$.

\section{(b) Concentration addition (CA)}

The typical application of this hypothesis avoids the formulation of an explicit response surface model. This surface is indirectly analyzed through isoboles, or projections of equal response lines on the plane of the independent variables (Berenbaum, 1985a; 1985b; Sørensen et al., 2007; Vølund, 1992). Although the criteria used by Berenbaum to define the null interaction can also be used to formulate an explicit model (Murado et al., 2011), the response to a mixed dose of two agents can be postulated as the response of two fictitious "mixed" doses of the same agent as follows:

$$
R=W\left[\left(A_{1}+A_{2}\right) ; K, m, a\right]
$$

Any interaction that is considered must preserve the key concept of the concentration addition, implying that the doses in equation (11) should act as an additive block within an algebraic expression with a single set of parameters $(K, m, a)$. Accordingly, the possible perturbations are as described below: 
- Different antioxidant power. The model is obtained by introducing a factor, $p$, to one of the doses ( $p<1$ if the affected antioxidant is the most powerful):

$$
R=W\left[\left(p A_{1}+A_{2}\right) ; K, m, a\right]
$$

Notably, this effect does not alter the condition of null interaction, and if a joint response can be described by the equation (12), the $m_{1}$ value of the individual response to $A_{1}$ is $m_{1}=m / p$.

- Interactions modifying the effective dose. If an antioxidant, $A_{1}$, reciprocally or non-reciprocally interacts with another $A_{2}$, in such a way that the effect of $A_{2}$ is equivalent to the effect due to an effective dose higher or lower than the nominal one, the different alternatives can be described by the following model, by using $v_{\theta \mathrm{i}}$ terms such as those $\left(v_{\mathrm{Ai}}\right)$ defined in $(8)$ :

$$
R=W\left[\left(A_{1} \frac{1+b_{1} A_{2}}{1+c_{1} A_{2}}+A_{2} \frac{1+b_{2} A_{1}}{1+c_{2} A_{1}}\right) ; K, m, a\right]
$$

- Interactions modifying the sigmoidal parameters. In general, the interactions in which each antioxidant specifically modifies the sigmoidal parameters $(K, m, a)$ of the joint response can be considered according to a model as follows:

$$
R=W\left[\left(A_{1}+A_{2}\right) ; K v_{k 1} v_{k 2}, m v_{m 1} v_{m 2}, a\right]
$$

Theoretically, this relationship implies that the individual responses increase non-asymptotically or decrease after a maximum (in the latter case with a similar profile to that produced by an enzymatic kinetic with substrate inhibition). Experimental evidence of this behavior has been found in the dose-response area (Cabo et al., 2000). However, either response is uncertain in the context of the interactive action of antioxidant agents. Nevertheless, the general model for $C A$ in its more complex form is defined as follows:

$$
R=W\left[\left(p A_{1} v_{A 1}+A_{2} v_{A 2}\right) ; K v_{k 1} v_{k 2}, m v_{m 1} v_{m 2}, a\right]
$$

As noted with respect to the model (10), most practical situations should be resolved with simpler particular cases.

\subsection{Numerical and statistical methods}

\subsubsection{Basic methods}

- Fitting procedure: simulated and experimental results were adjusted to the proposed models by non-linear least squares methods (quasi-Newton), using Solver complement.

- Parametric estimations: were performed by incorporating the 'SolverAid' macro (Prieto et al., 2012b; Prikler, 2009) for estimating the confidence intervals.

- Model consistency tests: student's $t$ and Fisher's $F$ tests, respectively, with $\alpha=0.05$ in both cases.

- Model selection criteria: Because there were many possible combinations of parameters able to fit the combined effects of both antioxidants, a selection process needs to be applied to determine the model that best predicts the joint effect of the two variables in the interval studied. Therefore, different model selection criteria (MSC) were used to evaluate the appropriateness of the equations. For more details about the process of selection and its pitfalls a specific description is presented in the appendix section (Table A1 and Table A2).

\subsubsection{Development of an automatic stepwise regression method for the analysis of the responses}

Although the initial number of parameter combinations (models with interactions (10) and (15)) is high, this number only signifies a high number of potential alternatives. The most complex cases that 
were found involved a maximum of four interactive parameters plus those for the individual responses ( 6 and 4 for the $I A$ and $C A$, respectively). However, in occasions when a large amount of data needs to be analyzed, the process of finding the most appropriate solution can be very laborious. Therefore, a stepwise regression method was developed by programming a routine in excel in which all possible parameter combinations are tested. The routine involved the following steps: 1) fitting the sigmoidal parameters from the individual responses (without interactions), using equation (7) and (11) for the $I A$ and $C A$ hypothesis, respectively; 2) these estimates were then used as the starting values for assaying all possible parameters combinations of the model (10) (IA, 9 parameters and 511 combinations) and (15) (CA, 13 parameters and 8.191 combinations); 3) rejecting the options that lead at least to a none statistically significant coefficient; and 4) selecting the most remarkable solutions, which are automatically ranked with several model selection criteria to differentiate the most "true solution".

\section{RESULTS}

\subsection{Meaning of the synergy and antagonism notions}

Once the equations (10) and (15) were accepted as generalized models for $I A$ and $C A$ hypotheses, respectively, an algebraic framework was established that characterizes synergy and antagonism through the specific variations imposed by the perturbations to the parameters and the response.

In a broad sense, an interaction is synergistic or antagonistic as it increases or decreases the expected response in the null interaction. In the $I A$ model (10), a synergistic interaction raises at least one $K_{\mathrm{i}}$ parameter, reduces at least one $m_{\mathrm{i}}$, reduces the $s$ coefficient or imposes all these effects simultaneously, while antagonism determines the opposite effects. In the $C A$ model (15), synergy and antagonism are translated into changes of the effective concentrations according to the equation (13), as well as, into variations of $K$ and $m$ parameters as in the $I A$ model, at least theoretically. Notably, the modification of the effective concentration according to equation (13) is not mathematically possible in the $I A$ model, in which the corresponding effect must be translated into variations of $m_{\mathrm{i}}$ parameters.

These definitions may be further restricted if the conventional analysis applied in toxicology to $C A$ model is accepted. As already mentioned, this model is assessed by the isobole examination, accepting that straight, concave up and convex up isoboles indicate a null interaction, synergy and antagonism, respectively. Because this behavior only occurs in the perturbations described by the equation (13), synergy and antagonism could be limited to the interactions modifying the effective concentrations.

However, this restriction does not logically follow for two reasons: 1) other effects may increase or decrease the response corresponding to the null interaction without altering the effective concentration, and these effects should not be excluded from the synergy and antagonism definitions; 2) the isobole approach is only applicable in the context of the $C A$ hypothesis, specifically, in cases that can be described by the equation (13). In fact, the complexity of the isoboles in the IA hypothesis prevents the use of the simple criterion of their concavity or convexity (cita Murado, PlosOne). Therefore, the concepts of synergy and antagonism will be used according to the broad sense defined before.

\subsection{A step by step example of the methodological process}

The methodological procedure and the mathematical models proposed in the previous sections yielded consistent results when combining all the antioxidants listed in the materials section for each of the methods. These results not only permitted the decision between the null interaction, synergy and antagonism, but also revealed some interesting aspects of the system used and the approach applied. To illustrate the methodological procedure of this approach, the joint action of TOC $\left(A_{1}\right)$ and BHA $\left(A_{2}\right)$ on the bleaching reaction of $\beta$-carotene will be described in detail (Figure 2, Figure 3 and Table $1)$.

\subsubsection{Procedure to obtain the $R A U$ values}


Figure 2 shows the procedure to obtain the $R A U$ responses using the TOC $\left(A_{1}\right)$ and BHA $\left(A_{2}\right)$ antioxidant combination in the $\beta$-carotene reaction as an example. Figure $2 \mathrm{~A}$ shows the remaining raw responses of the substrate ( $\mathrm{SH}$, in this case $\beta$-carotene in $\mu \mathrm{M}$ ) for the reaction in the presence of TOC and BHA. In each single graph of the $8 \times 8$ array, the top line shows the response for the control, the bottom line shows the response for the corresponding combination of antioxidants and the shadow area shows the $R U A$ values. In Figure $2 \mathrm{~B}$ presents the obtained $R U A$ data first in two separated $2 \mathrm{D}$ graphs that show the response in a non-standardized form as the individual effects caused for each antioxidant, and then as the response and antioxidant doses that are standardized to a scale of $[0,1]$ presented in a single 3D graph.

Once the $R A U$ responses were obtained, the modeling procedure to determine, characterize and quantify the interactive effects could be started. The procedure will be performed in different ways, first by analyzing intuitively the possible modes of action with and without interactions and afterwards applying the automatic stepwise regression method developed. The findings below demonstrate that both criteria converge into identical solutions.

\subsubsection{Intuitive analysis of the hypothetical modes of action with and without interactions}

(a) TOC (A1) and BHA (A2) assuming independent action (IA)

The null interaction in the $I A$ hypothesis implies that the joint action should be described by adjusting the individual responses to the model (7) and using the obtained parameters in the model (10), with all $v_{\theta \mathrm{i}}=1\left(b_{\theta \mathrm{i}}=c_{\theta \mathrm{i}}=0\right)$ and $s=1$. By proceeding in this way, the $r^{2}$ and $R_{\text {adj }}^{2}$ values, as well as the Student's $t$ and Fisher's $F$ test (both with $\alpha=0.05$ ) applied to the parametric estimations and to the explained variance, respectively, showed a statistically acceptable fit (Figure 3 and Table 1). However, the distribution between the observed and predicted results (OP) was biased, and the residuals showed that the computed response surface predicts lower values than those experimentally obtained, which suggests a synergistic interaction.

Indeed, a decrease in the OP bias and an improvement in the other fitting criteria were obtained by accepting a drop in the $m$ parameter of the response to TOC due to the presence of BHA (increasing antioxidant potency: synergy in the strict sense). A further improvement could be obtained by accepting a similar drop in the $K$ parameter (antagonism in the broad sense, less strong than the synergistic effect). Although the interactions producing simultaneous opposite effects on the response are in general neither formal nor mechanistically rejectable, the predicted individual responses in this case are statistically less correct than those corresponding to the simpler hypothesis of synergy. The decision would probably be clearer by slightly expanding the experimental domain, which could more precisely define the asymptotes of the individual responses. Nevertheless, the net effect of the interaction between TOC and BHA is synergistic.

\section{(b) TOC (A1) and BHA (A2) assuming concentration addition (CA)}

Under the $C A$ hypothesis, the null interaction requires to set all $v_{A i}=v_{\theta i}=1$ in model (15). When the relative potency coefficient $(p \neq 1)$ was included under these conditions, the model produced a statistically significant description $\left(r^{2}, R^{2}{ }_{a d j}, t\right.$ and $\left.F\right)$. However, a biased OP distribution and residuals indicating a general underestimation of the predicted response with respect to the experimental results were again obtained (Figure 3 and Table 1). All fitting criteria improved significantly when a synergistic effect (strict sense) was included (one of the antioxidants increases the effective concentration of the other, a situation in which the $C A$ model cannot distinguish directionality). If a hyperbolic variation of the effective concentrations was assumed, the fit was slightly higher than that corresponding to a linear variation. However, the correlation between the coefficients of the perturbation term penalizes their confidence intervals, which confines such coefficients close to the lack of statistical significance. 
After considering these intuitive options, the description obtained by supposing linear variations of the effective doses under $C A$ hypothesis was more accurate than those found under $I A$ alternative. Therefore, it must be concluded that the model of the joint response to TOC and BHA obeys the CA mode with synergistic interaction.

\subsubsection{Automatic analysis by a stepwise regression method}

When a large set of data needs to be analyzed, the intuitively process of finding the most appropriate solution can be very laborious. Therefore, an automatic procedure that integrates a set of statistical MSC to rank and selected the most appropriate solution has been developed by programming a routine in excel in which all possible parameter combinations were tested. For example, the top fitting results of TOC and BHA case for each mode of action after applying this automatic system to evaluate the results are shown in Table A2 (appendix section). The data demonstrates that the overall best model was the $C A$ hypotheses, and within this hypothesis the case number 4 predicts accurately the data, being the most likely response to be correct. This selection was identical to that intuitively found above, which demonstrates the reliability of both ways for selecting the correct solution. However, because the automatic system is undoubtedly faster and reliable, it was the procedure used to assess all pairs of tested antioxidants.

\subsection{Other findings drawn from the analysis of the joint action}

Certainly, the solution can be directly determined in the majority of the cases analyzed. However, both models provide equal satisfactory results in occasions, such as when the solution is a continuum or mixed response of both hypotheses showing difficulties to choose any as the correct solution. This ambiguity has also been found in a recent and extensive revision (158 items) of experimental results carried out by Cedergreen et al. (2008). The consequences of our approach agree with those findings.

In addition to the statistically consistent detection of the interactive effects, for example, the analysis of TOC and BHA raises a more basic question related to the status of $I A$ and $C A$ hypotheses. The experimental results were better described by $C A$ than by $I A$ hypotheses. TOC and BHA could be accepted that act at the same point of a general oxidative pathway as summarized in Figure 1. Irrespective of this comparison, the description under IA hypothesis could not be rejected by applying common statistical criteria, which implies that the antioxidants act at different points of that pathway.

This ambiguity can be explained in terms of the relationships between the rate constants involved in the reaction sequence of the mentioned pathway. Considering Figure 1, it can be admitted that if the activity of the antioxidant act only through the $k_{1}$ and $k_{4}\left(k_{2}=k_{3}=0\right)$, or $k_{2}$ and $k_{3}\left(k_{1}=k_{4}=0\right)$ pathways, the model is $I A$, and if only acts through the $k_{1}$ and $k_{3}\left(k_{2}=k_{4}=0\right)$, or $k_{2}$ and $k_{4}\left(k_{1}=k_{3}=0\right)$ pathways, the model is $C A$. However, other less extreme situations clearly take place in which none of the rate constants equal zero. If the pathways $k_{1}$ and $k_{4}$ or $k_{2}$ and $k_{3}$ are simply dominant $\left(k_{1}>k_{2}\right.$ and $k_{4}>k_{3}$, or $k_{1}<k_{2}$ and $k_{4}<k_{3}$ ), the model will be predominantly $I A$; and if the dominant mechanisms are $k_{1}$ and $k_{3}$ or $k_{2}$ and $k_{4}\left(k_{1}>k_{2}\right.$ and $k_{3}>k_{4}$, or $k_{1}<k_{2}$ and $\left.k_{3}<k_{4}\right)$, the model will be predominantly $C A$. Different mechanism of each antioxidant in the convergence points 1 and 2 of Figure 1 also serve as contributions to ambiguity.

Therefore, the joint antioxidant effect of TOC and BHA on the linoleic acid/ $\beta$-carotene system strongly suggests a predominant action on the same point of the oxidative sequence, without implying that the antioxidants act only at one point.

A further achievement of these results is the presentation of the $I A$ and $C A$ hypotheses as the two ends of the same continuum, contrary to their usual presentation as mutually exclusive options. At the extremes, only one of the hypotheses will produce a statistically acceptable result; cases in which both 
hypotheses are consistent exist within the continuum. In this case, the selection of the hypothesis that provides the best solution does not imply a lack of contribution of the other hypothesis.

If the relatively simple context of an in vitro antioxidant action provides ambiguous cases determined by the relationships between reaction rates of a schematic sequence, similar ambiguities necessarily and probably arise in the field of in vivo dose-response relationships (Murado et al., 2011). This last field has been noted (Jonker et al. 2005) to provide cases that do not follow any of the two classical hypotheses. We believe that the preceding results explain why reality exhibits cases in which both hypotheses are simultaneously obeyed.

\subsection{Other examples of joint action that illustrate important aspects}

By applying the methodological procedure to the joint action of several pairs of antioxidants, all solutions were described by one of the models or by both. However, not less important issues are occasionally found. Next, some of these aspects are confronted and discussed in detail.

\subsubsection{Need for additional criteria to assist the selection process: $A A\left(A_{1}\right)$ and ETX $\left(A_{2}\right)$ in the crocin reaction}

This is a typical case in which the selection of the mode of action it was less questionable, but the selection of the interactive effects was complex, and it needs a deeper analysis that uses intuitive criteria to select the most correct solution. As in the case of TOC and BHA, the null interaction was not acceptable in any of the two modes of action, and the residuals suggested a synergistic effect. Contrary to what has been found in the previous case, the worst fitting solutions were obtained with $C A$ model (15), and the best ones were provided by the IA model (10), in which three preliminarily acceptable possibilities can be found:

a) $\quad A_{2}$ reduces the parameter $m$ of the response to $A_{1}$ (synergy in the strict sense).

b) $A_{2}$ increases the parameter $K$ of the response to $A_{1}$ (synergy in the broad sense).

c) Generic cooperative action $(s<1)$ between antioxidants (synergy in the broad sense).

The confidence intervals (CI) of parameters yield to prefer the $a$ option rather than the other two strict sense synergistic forms (unidirectional opposite and reciprocal), and the OP and $R_{a d j}^{2}$ criteria allowed the rejection of option $b$. The decision between $a$ and $c$ was uncertain, because $c$ produces a better fit, but it generates an excessive effect, which produces responses higher than 1.03 in a small subdomain of simultaneous high concentrations of both antioxidants. Although the subdomain and deviation are of scarce importance, the less global option, $a$, does not create this problem, narrows the confidence intervals (CI) and reduces the fitting only slightly. Due to any combination of $a, b$ and $c$ did not produce acceptable results, option $a$ seemed to be finally the best solution.

In other words, the results indicated a predominantly independent action that was clearly synergistic, which suggests the following: 1) at least one of the antioxidants acts at two different points in the oxidation sequence of the crocin reaction; 2) at one of these points, the action of the other antioxidant can be neglected; and 3) at the other point, where both antioxidants act through the same mechanism by adding their concentrations, the antioxidant effect is poor.

\subsubsection{Antagonistic effects in the framework of the antioxidant action: $M n\left(A_{1}\right)$ and $A A\left(A_{2}\right)$ in crocin reaction}

One important question in the join action of two or more antioxidants is related to the possibility of obtaining combinatory responses lower than the expected responses of their individual effects, or in other words antagonistic effects. The interactive activity between $\mathrm{Mn}\left(A_{l}\right)$ and AA $\left(A_{2}\right)$ in the oxidation of crocin is a clear example of such a case. When both antioxidants are tested independently, they show a clear antioxidant character. However, when combined, the Mn significantly depressed the 
effect of AA, which continuously decreased the maximum response (the parameter $K_{2}$ ). The joint response could be broadly described in a statistically significant way as an IA case with an antagonistic effect.

If transferable to real systems, such as the preservation of food and beverages in hydrophilic surroundings, these results indicate that the presence of $\mathrm{Mn}$, a typical compound in plants, will diminish the activity AA, a typical antioxidant in hydrophilic environments, which would reduce the expected life of the system.

\subsubsection{Cooperative effects: $A A\left(A_{1}\right)$ and $T R O\left(A_{2}\right)$ in crocin reaction}

Both models (IA and $C A$ ) indicated a synergistic (strict sense) joint response, with statistically higher results for the $I A$ option. In this case, the fit improves if the synergy is complemented by a slight generic cooperative effect $(s<1)$, which defines the response between AA and TRO as predominantly $I A$ with antagonistic effects. As in the previous case, these results would indicate significant issues in different disciplines of food science in real systems.

\subsubsection{Low response effects for one of the agents: TRO (A1) and BHT (A2) in $\beta$-carotene reaction}

This case represents an example of the "polar paradox" (Frankel et al., 1994; 2005; Koleva et al., 2002; Porter, 1993), a typical phenomenon in lipid emulsion systems, such as the $\beta$-carotene reaction. It favors the activity of the non-polar antioxidants over the activity of polar antioxidants, because the hydrophobic repulsion tends to concentrate the first non-polar antioxidants (i.e., BHT), but not the polar ones (i.e., Trolox) in the lipid environment where the oxidation occurs. In fact, the Trolox activity was very low in the concentration domain tested, showing a linear profile, an imperceptible contribution to the joint response at high levels of BHT. This linear relationship causes linear correlations between the coefficients of the perturbation terms $\left(v_{\theta i}\right)$ and penalizes the CI of the parametric estimations, which increases as the experimental error increases and as the number of observations decreases. This low response is one weakness of models (10) and (15). Fortunately, accurate data are effortlessly obtained by working with microplate readers, and both problems are thus minimized.

The description of the system was statistically significant assuming $I A$ when BHT reduces the $m$ parameter of the response to Trolox. Therefore, the joint response was broadly typified as an independent action case with an antagonistic effect.

\subsection{In search of a comprehensive index}

If a single numerical value that summarizes the nature and the intensity of the synergistic or antagonistic interactions could be proposed, this clearly would help and become a useful index in different scientific fields. Once an explicit algebraic model for a response surface is settled, the definition of such an index seems to require only a comparison between the response corresponding to the null interaction hypothesis and the experimentally obtained response. The usefulness of this approach from a theoretical and practical perspective is questionable. In fact, neither the difference nor the quotient between the typical responses in null interactions and any interactive situation remains constant throughout the domain of the independent variables (see Figure 5). Thus, any index that is calculated at a specific point (e.g. for $A_{1}=m_{1}$ and $A_{2}=m_{2}$ ), or along a specific response (e.g. the halfmaximal response), cannot account what happens in another region of the response surface. This fact is true even in a simple case as $s \neq 1$ in model (10), and specific situations can exist (as opposite variations in $K_{\mathrm{i}}$ and $m_{\mathrm{i}}$ parameters) in which the net effect is synergistic in one subdomain of the response surface and antagonistic in another one.

However, in an effort to find a comprehensive index, the best alternative to summarize such a response could be to compute the percentage relative unit of volume $(R U V)$ between the volume of the surface 
produced by the null interaction $\left(S V_{N I}\right)$ and the volume of the surface with interactions $\left(S V_{I}\right)$ as follows:

$$
R U V=\frac{S V_{I}-S V_{N I}}{S V_{I}} \times 100 ; \text { being } S V=h_{i} h_{j} \sum_{i=0}^{n} \sum_{j=0}^{m} f\left(A_{i} A_{j}\right) \phi_{i, j}
$$

in which $A_{\mathrm{i}}$ and $A_{\mathrm{j}}$ are the dependent variables that represent the $n$ and $m$ concentration of both antioxidants, $h_{\mathrm{i}}$ and $h_{\mathrm{j}}$ are the concentration interval sets and $\Phi_{\mathrm{i}, \mathrm{j}}$ is the product of the nested composite trapezoidal rule coefficients. Therefore, positive and negative values of $R U V$ will describe the predominantly synergistic and antagonistic interaction effects between the antioxidants over the study range.

The variations in the parametric values of the response to an antioxidant as a function of the concentration of the other antioxidant (the structures of the perturbation terms) or the global approach of computing the $R U V$ allow a brief reasonable description of the interactive effects. However, because the datum of practical interest is the possible difference between the null interaction and the experimental result in a given domain, only the "scenery" of these differences throughout the experimental domain allows effective and statistically founded statements.

The results for the $R U V$ obtained for all 42 cases assessed are presented in Table 3. The full analysis of all the possible combinations is presented in the appendix (Figure A2, Figure A3, Table A3 and Table A4).

\section{DISCUSSION}

Synergy and antagonism are controversial characteristic behaviors of very diverse systems. Despite their importance, the common characterization of these phenomena in the context of the antioxidant action is often questionable due to some problematic definitions and the type of data used. The models proposed here showed a good ability to describe the joint action of several pairs of antioxidant under both aqueous and lipid emulsions. Their application allows to: 1) typify a joint antioxidant activity in terms of the two modes of joint action accepted in the field of dose-response relationships; and 2) led to the detection and quantification of synergistic and antagonistic effects by comparing, for each mode of action, the fitting of the experimental results to several formal models by describing different interaction scenarios, including null interaction.

Additionally, the results have proven that: 1) when synergy and antagonism are defined in the broad sense as interactions increasing or decreasing the response corresponding to the null interaction, several modalities of those effects arise, depending on the mode of action considered and the parameters of the response to an antioxidant which are modified by the presence of the other one; 2 ) synergistic and antagonistic consequences can vary along the response surface, even effects with the opposite signs in different subdomains of that surface are produced; 3) insofar as independent action and concentration addition models define -in very general terms- mechanisms, it is possible to connect the different forms of the models (10) and (15) with equally general aspects of the mechanisms involved in the oxidative pathways; 4) under this last perspective, $I A$ and $C A$ hypotheses arise -in opposition to the common idea of mutually exclusive possibilities - as the two extremes of a continuum. Such a continuum is characterized by the sites in which a given oxidative pathway is inhibited, and the relations between the rate constants of the inhibitory reactions (Figure 1).

\section{CONCLUSIONS}

In this paper, a methodological procedure has been developed for the joint action of several pairs of antioxidants in both aqueous and lipid emulsions, which enables the determination and quantification of the synergistic and antagonistic interactive effects. Although the approach could be directly 
expanded to other types of classical antioxidant methods, the methods selected are fairly representative of the most complex scenarios that can be found in the oxidation process. Unfortunately, the proposed approach is a little more complex than some relatively common solutions appearing in the bibliography. However, we believe that it is free of the most controversial aspects of such solutions.

\section{ACKNOWLEDGEMENTS}

The authors wish to thank CSIC (Intramural project: 200930I183) and Ministerio de Ciencia e Innovación (project CTM2010-18411, co-financed with FEDER funds by European Union) for financial support. Miguel Angel Prieto Lage was awarded one grant from the JAE predoctoral program co-financed by the CSIC and European Social Fund (ESF). 


\section{MANUSCRIPT REFERENCES}

Baldwin, W. S., \& Roling, J. A. (2009). A concentration addition model for the activation of the constitutive androstane receptor by xenobiotic mixtures. Toxicological sciences: an official journal of the Society of Toxicology, 107(1), 93-105.

Berenbaum, M. C. (1985a). Consequences of synergy between environmental carcinogens. Environmental research, 38(2), 310-318.

Berenbaum, M. C. (1985b). The expected effect of a combination of agents: the general solution. Journal of theoretical biology, 114(3), 413-431.

Bliss, C. I. (1939). The toxicity of poisons applied jointly. Annals of applied biology, 26(3), 585-615.

Bliss, C. I. (1937). The calculation of the time-mortality curve. Annals of Applied Biology, 24, 815824.

Bors, W., Michel, C., \& Saran, M. (1984). Inhibition of the bleaching of the carotenoid crocin a rapid test for quantifying antioxidant activity. Biochimica et Biophysica Acta (BBA) - Lipids and Lipid Metabolism, 796(3), 312-319.

Cabo, M. L., Murado, M. A., González, M. P., \& Pastoriza, L. (2000). Dose-response relationships. A model for describing interactions, and its application to the combined effect of nisin and lactic acid on Leuconostoc mesenteroides. Journal of applied microbiology, 88(5), 756-763.

Dávalos, A., Gómez-Cordovés, C., \& Bartolomé, B. (2004). Extending Applicability of the Oxygen Radical Absorbance Capacity (ORAC-Fluorescein) Assay. Journal of Agricultural and Food Chemistry, 52(1), 48-54.

Frankel, E. N. (2005). Lipid Oxidation. The Oily Press (pp. 2865).

Frankel, E. N., Huang, S. -., Kanner, J., \& Bruce German, J. (1994). Interfacial phenomena in the evaluation of antioxidants: Bulk oils vs emulsions. Journal of Agricultural and Food Chemistry, 42(5), 1054-1059.

Gessner, P. K. (1988). A straightforward method for the study of drug interactions: an isobolographic analysis primer. International journal of toxicology, 7, 987-1012.

Greco, W. R., Bravo, G., \& Parsons, J. C. (1995). The search for synergy: a critical review from a response surface perspective. Pharmacological reviews, 47(2), 331-385.

Hewlett, P. S., \& Plackett, R. L. (1964). A unified theory for quantal responses to mixtures of drugs: competitive action. Biometrics, 20(3), 566-575.

Huang, D., Ou, B., Hampsch-Woodill, M., Flanagan, J. A., \& Prior, R. L. (2002). High-Throughput Assay of Oxygen Radical Absorbance Capacity (ORAC) Using a Multichannel Liquid Handling System Coupled with a Microplate Fluorescence Reader in 96-Well Format. Journal of Agricultural and Food Chemistry, 50(16), 4437-4444.

Huang, Y. B., Yang, S., Cai, Y., Wang, H., Gao, G., Wu, X. X., Xu, L., \& Zhang, X. Z. (2008). Alcalase-catalysed synthesis of the precursor tetrapeptide N-benzoylarginylglycylaspartylserinamide 
(Bz-RGDS-NH2) of the cell-adhesion peptide arginylglycylaspartylserine (RGDS). Biotechnology and applied biochemistry, 51(Pt 3), 119-127.

Jia, Z., Zhou, B., Yang, L., Wu, L., \& Liu, Z. (1998). Antioxidant synergism of tea polyphenols and small alpha]-tocopherol against free radical induced peroxidation of linoleic acid in solution. Journal of the Chemical Society, Perkin Transactions 2, (4), 911-916.

Jonker, M. J., Svendsen, C., Bedaux, J. J. M., Bongers, M., \& Kammenga, J. E. (2005). Significance testing of synergistic/antagonistic, dose level-dependent, or dose ratio-dependent effects in mixture dose-response analysis. Environmental toxicology and chemistry, 24(10), 2701-2713.

Koleva, I. I., Van Beek, T. A., Linssen, J. P. H., De Groot, A., \& Evstatieva, L. N. (2002). Screening of plant extracts for antioxidant activity: A comparative study on three testing methods. Phytochemical Analysis, 13(1), 8-17.

Labuza, T. P., \& Dugan, L. R. (1971). Kinetics of lipid oxidation in foods. CRC Critical Reviews in Food Technology, 2(3), 355.

Loewe, S., \& Muischnek, H. (1926). Ãœer Kombinationswirkungen. Naunyn-Schmiedebergs Archiv fÃ̄/4r experimentelle Pathologie und Pharmakologie, 114(5-6), 313-326.

Marco, G. J. (1968). A rapid method for evaluation of antioxidants. Journal of the American Oil Chemists $\tilde{A} \phi \hat{a}, \neg \hat{a},, \phi$ Society, 45(9), 594-598.

Marinova, E., Toneva, A., \& Yanishlieva, N. (2008). Synergistic antioxidant effect of $\alpha$-tocopherol and myricetin on the autoxidation of triacylglycerols of sunflower oil. Food Chemistry, 106(2), 628633.

Miller, H. E. (1971). A simplified method for the evaluation of antioxidants. Journal of the American Oil Chemists Society, 48(2), 91-91.

Murado, M. A., \& Vázquez, J. A. (2010). Mathematical model for the characterization and objective comparison of antioxidant activities. Journal of Agricultural and Food Chemistry, 58(3), 1622-1629.

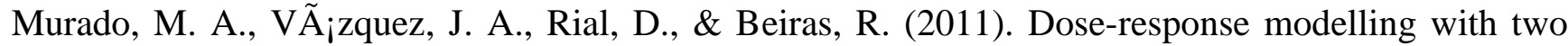
agents: application to the bioassay of oil and shoreline cleaning agents. Journal of hazardous materials, 185(2-3), 807-17.

Naguib, Y. M. A. (2000). A Fluorometric Method for Measurement of Oxygen Radical-Scavenging Activity of Water-Soluble Antioxidants. Analytical Biochemistry, 284(1), 93-98.

Özilgen, S., \& Özilgen, M. (1990). Kinetic Model of Lipid Oxidation in Foods. Journal of Food Science, 55(2), 498-498.

Parker, T. L., Miller, S. A., Myers, L. E., Miguez, F. E., \& Engeseth, N. J. (2010). Evaluation of synergistic antioxidant potential of complex mixtures using oxygen radical absorbance capacity (ORAC) and electron paramagnetic resonance (EPR). Journal of Agricultural and Food Chemistry, 58(1), 209-217.

Porter, W. L. (1993). Paradoxical behavior of antioxidants in food and biological systems. Toxicology and industrial health, 9(1-2), 93-122.

Prieto, M. A., Murado, M. A., Vázquez, J. A., Anders, Y., \& Curran, T. P. (2013). A new microplate procedure for simultaneous assessment of lipophilic and hydrophilic antioxidants and pro-oxidants, 
using crocin and $\beta$-carotene bleaching methods in a single combined assay: Tea extracts as a case study. Food Research International, 53(2), 836-846.

Prieto, M. A., Rodríguez-Amado, I., Vázquez, J. A., \& Murado, M. A. (2012). ß-Carotene Assay Revisited. Application To Characterize and Quantify Antioxidant and Prooxidant Activities in a Microplate. Journal of Agricultural and Food Chemistry, .

Prieto, M. A., Vázquez, J. A., \& Murado, M. A. (2012a). Crocin bleaching assay revisited. Application to characterize and quantify antioxidant and prooxidant activities in a microplate.

Prieto, M. A., Vázquez, J. A., \& Murado, M. A. (2012b). Comparison of several mathematical models for describing the joint effect of temperature and ph on glucanex activity. Biotechnology progress, 28(2), 372-381.

Prikler, S. (2009). Robert de Levie: Advanced Excel for scientific data analysis, 2nd ed. Analytical and Bioanalytical Chemistry, 395(7), 1945.

Qin, L. T., Liu, S. S., Zhang, J., \& Xiao, Q. F. (2011). A novel model integrated concentration addition with independent action for the prediction of toxicity of multi-component mixture. Toxicology, 280(3), 164-172. Rovati, G. E., \& Nicosia, S. (1994). Lower efficacy: interaction with an inhibitory receptor or partial agonism? Trends in pharmacological sciences, 15(5), 140-4.

Sørensen, H., Cedergreen, N., Skovgaard, I. M., \& Streibig, J. C. (2007). An isobole-based statistical model and test for synergism/antagonism in binary mixture toxicity experiments. Environmental and Ecological Statistics, 14(4), 383-397.

Terpinc, P., \& Abramovič, H. (2010). A kinetic approach for evaluation of the antioxidant activity of selected phenolic acids. Food Chemistry, 121(2), 366-371.

Vølund, A. (1992). Dose response surface bioassay. , In XVth Biometric Conference, vol II., p. 349-In XVth Biometric Conference, vol II., p. 349.

Weibull, W. (1951). A statistical distribution function of wide applicability. Journal of Applied Mechanics, 18, 293-297.

Yang, W. J., Li, D. P., Li, J. K., Li, M. H., Chen, Y. L., \& Zhang, P. Z. (2009). Synergistic antioxidant activities of eight traditional Chinese herb pairs. Biological \& pharmaceutical bulletin, 32(6), 10211026. 


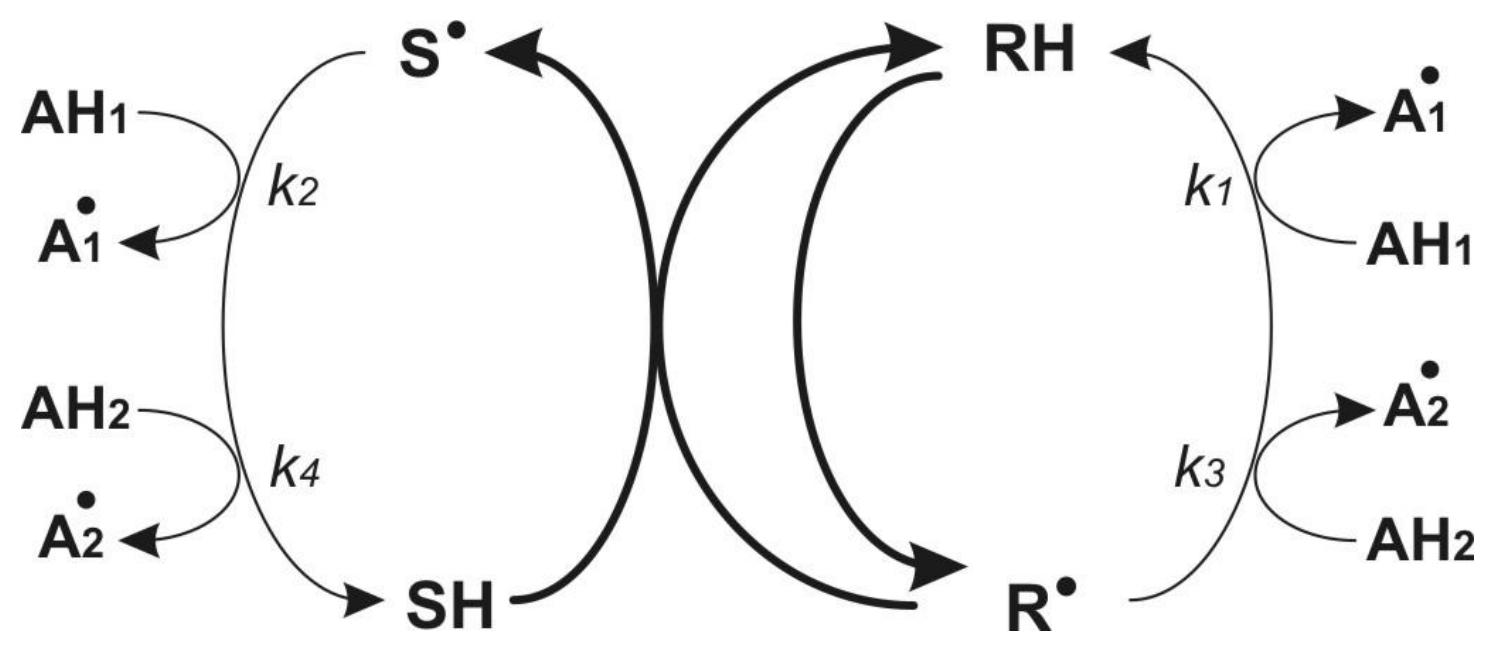

Figure 1: Oxidation of a SH substrate by $\mathrm{R} \bullet$ radicals from a $\mathrm{RH}$ source, in the presence of $\mathrm{AH}_{1}$ and $\mathrm{AH}_{2}$ antioxidants. Reactions $k_{1}$ to $k_{4}$ hinder the main pathway (tick lines). It is supposed that reactions $k_{1}$ and $k_{2}$ have the same mechanism, which is different of the one of the reactions $k_{3}$ and $k_{4}$. Under these conditions, the appropriate model for the antioxidant joint action depends on the relative values of the rate constants $k_{1}$ to $k_{4}$ (see text). 


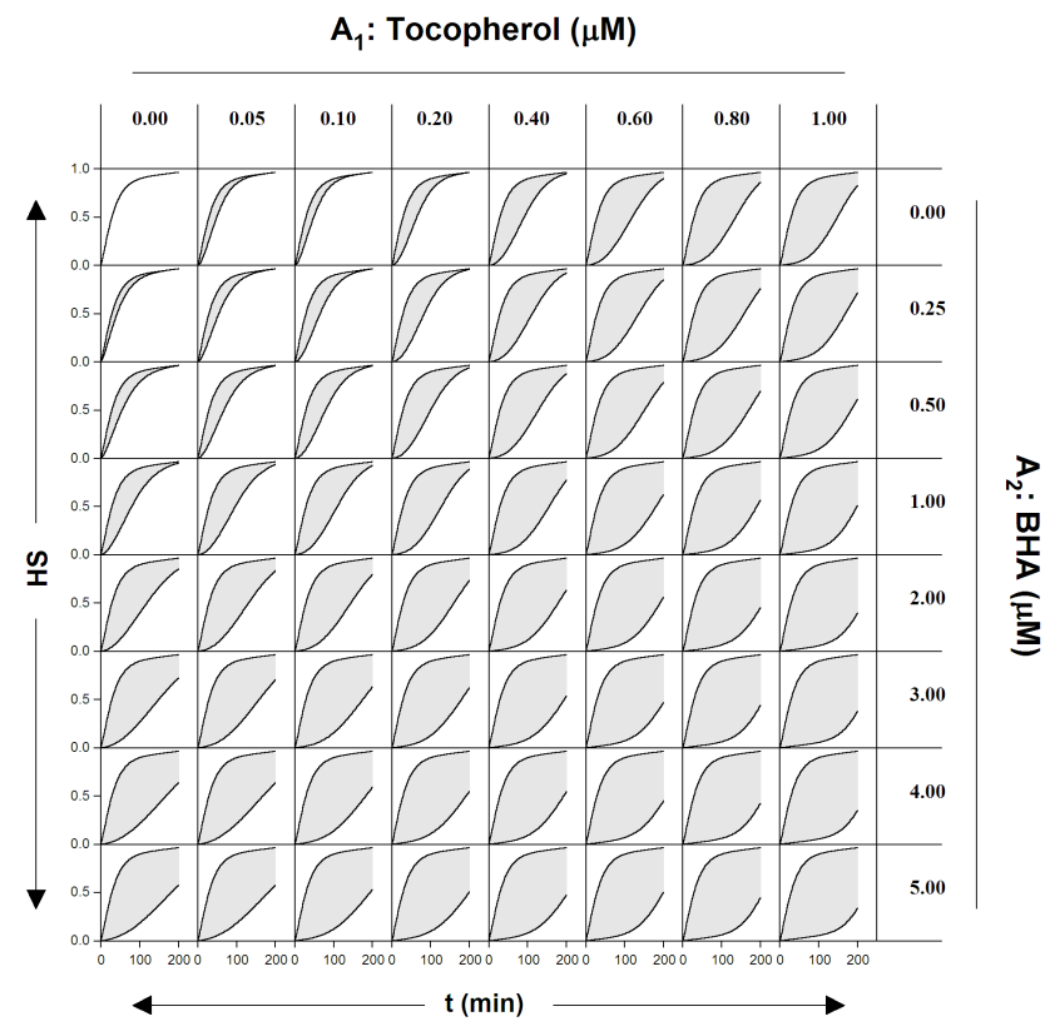

\section{B: RESPONSE AS RELATIVE AREA UNITS (RAU)}

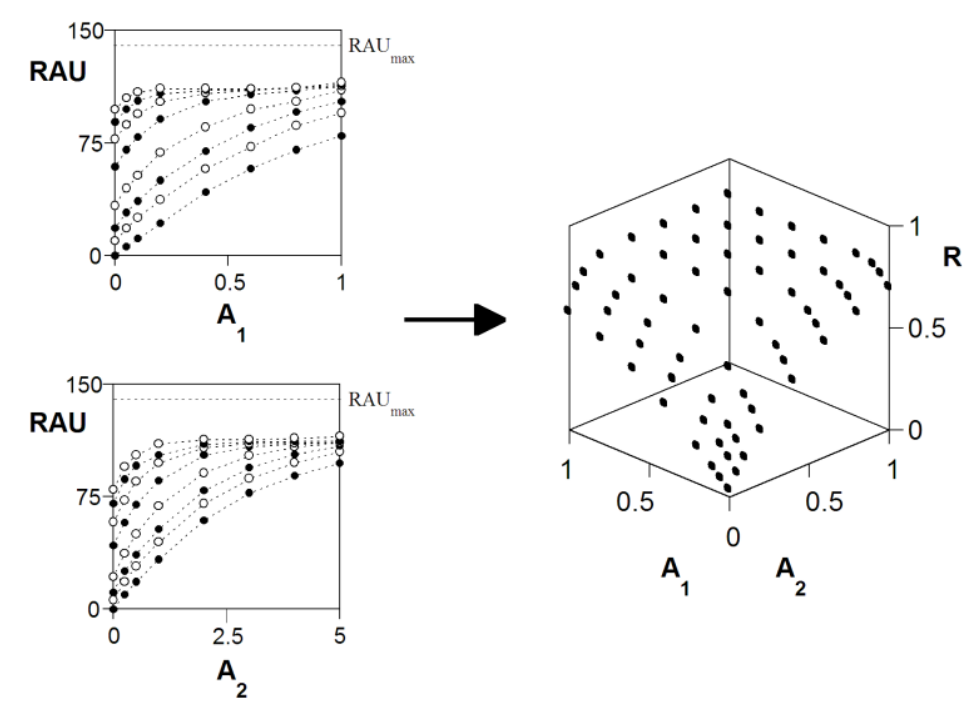

Figure 2: A descriptive example performed in stepwise mode, to show the process of obtaining the $R A U$ responses. $A$ : raw responses as remaining substrate (HS, in this case $\beta$-carotene in $\mu \mathrm{M})$ of the reaction in the presence of TOC $\left(A_{1}\right)$ and BHA $\left(A_{2}\right)$. In each single graph for the $8 \times 8$ array, the top line shows the response for the control, the bottom line the response for the corresponding combination of antioxidants and the shadow area the RUA values. $B$ : The obtained $R U A$ data is presented, first, in two 2D graphs with non-standardized response, showing the individual effects caused for each antioxidant. Afterwards, response and antioxidant doses are standardized to a scale $[0,1]$ and presented in a single $3 \mathrm{D}$ graph. 

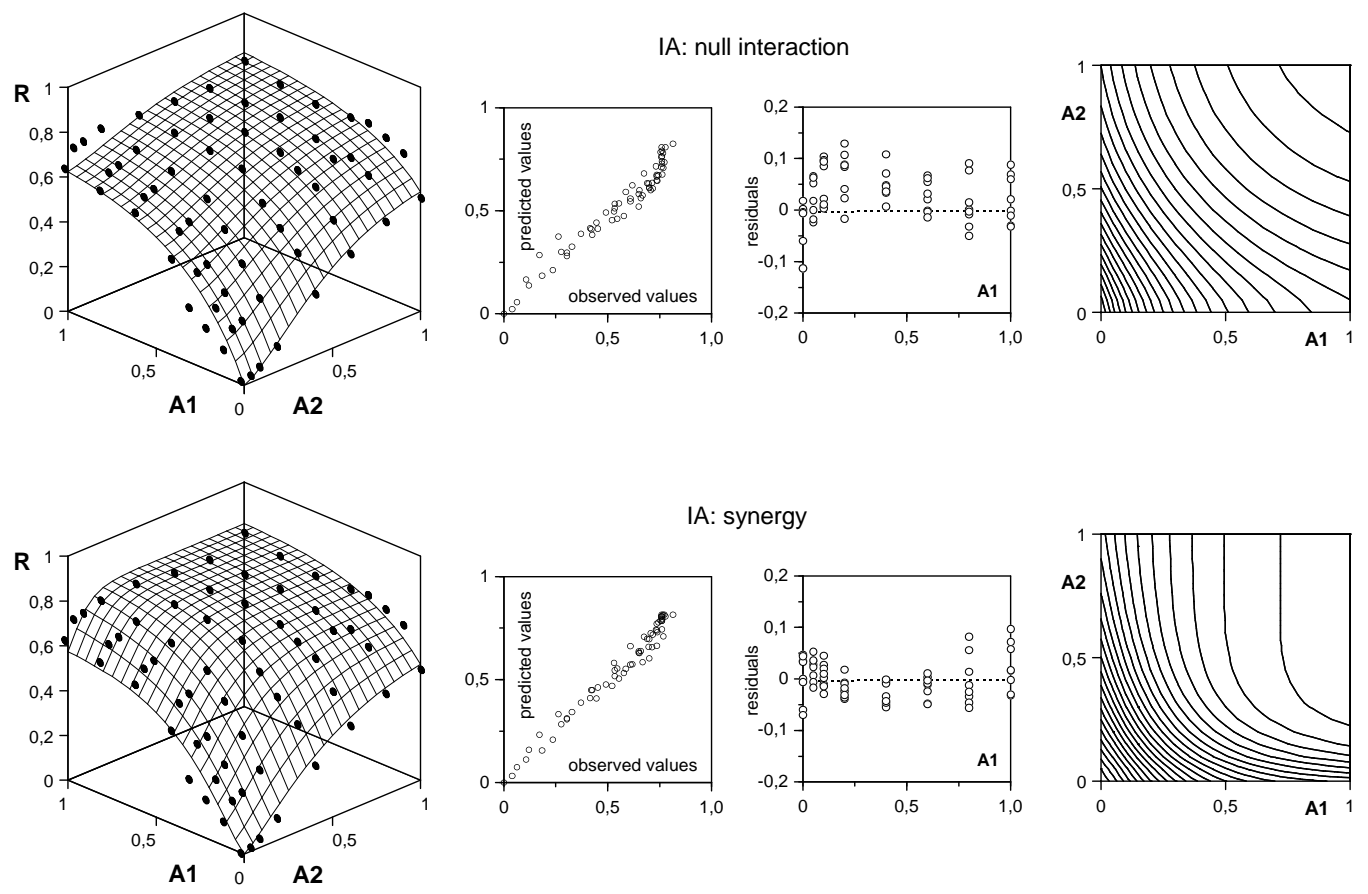

: synergy
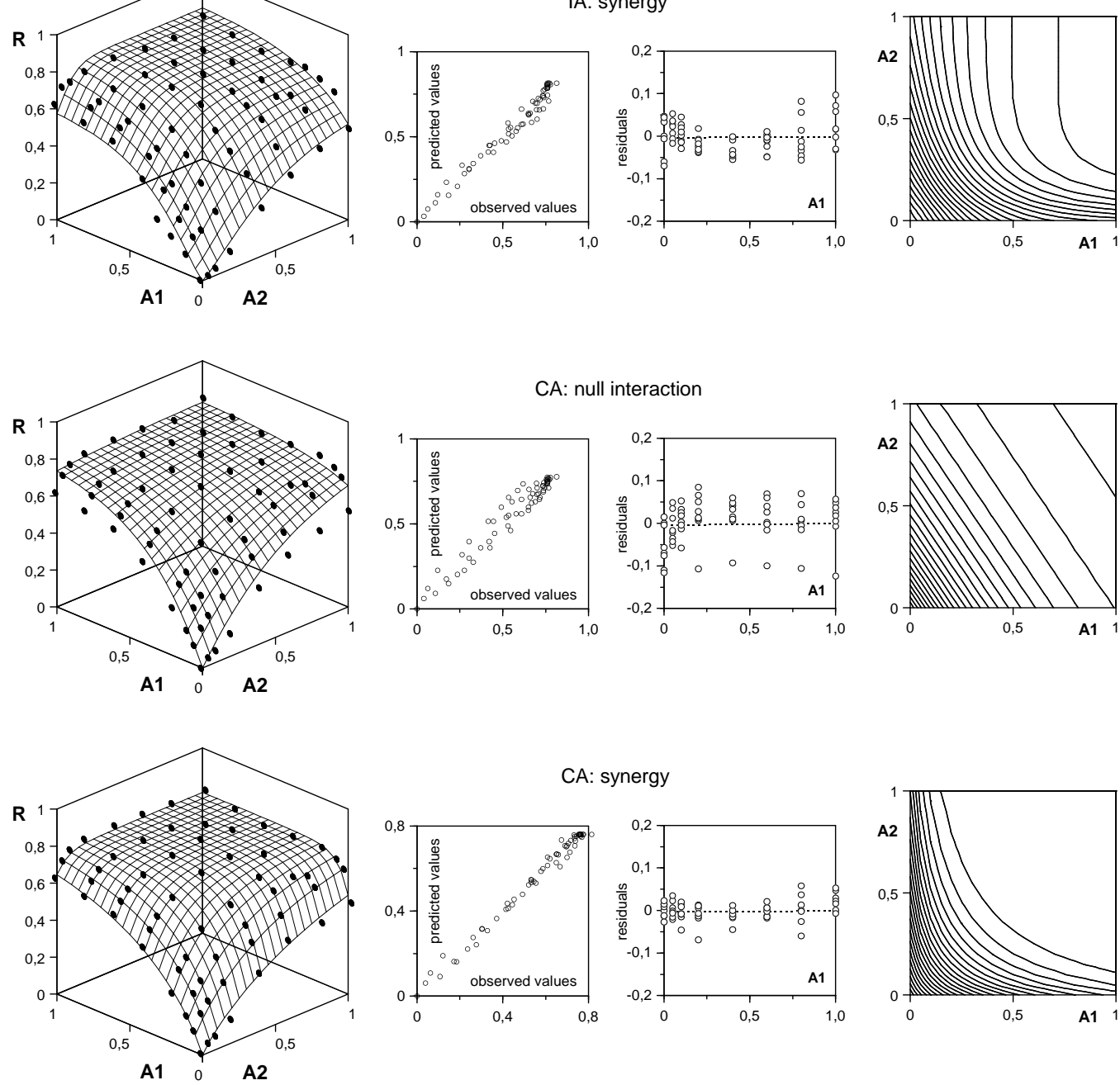

A: synergy
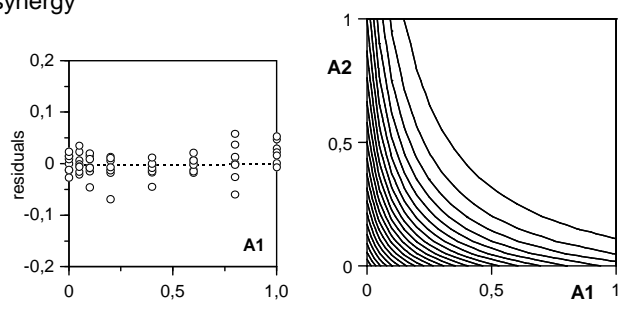

Figure 3: Joint effect of TOC $\left(\mathrm{A}_{1}\right)$ and BHA $\left(\mathrm{A}_{2}\right)$ on $\beta$-carotene oxidation under different hypotheses. Experimental results (points) and fittings to the models (10) and (15) (surfaces). Correlations between observed and predicted values, residuals and isobole projections of the response surfaces are also shown. See text for details. Numerical results in Table 1. 

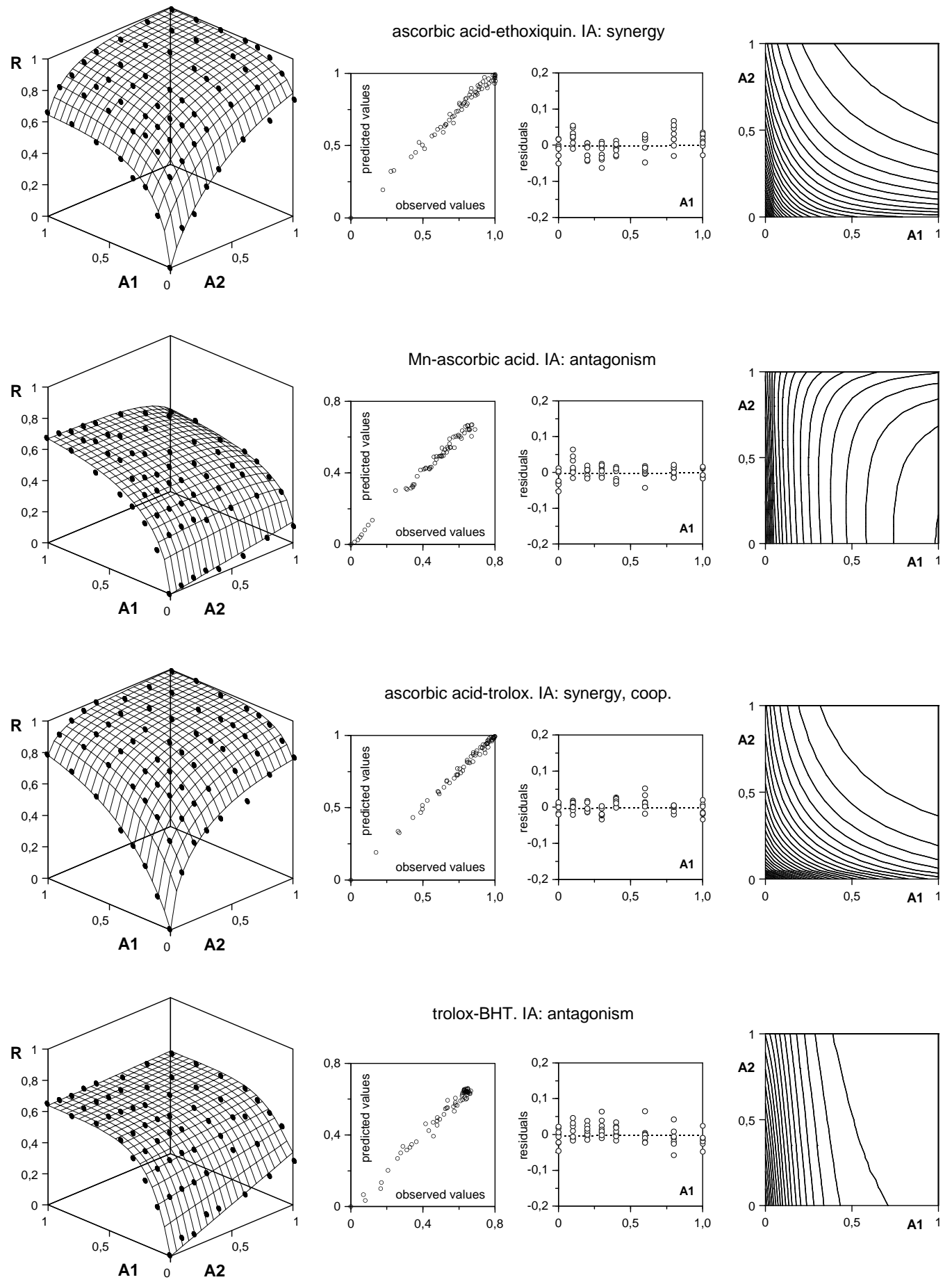

Figure 4: Characterization of the joint effect of the specified antioxidant pairs, using $\beta$-carotene (Trolox-BHT) and crocin (the rest) reactions. Graphic criteria and notations as in Figure 3. In ascorbic acid-Trolox, coop. means general cooperative action $(s<1$ in equation (10)). See text for details. Numerical results in Table 2. 


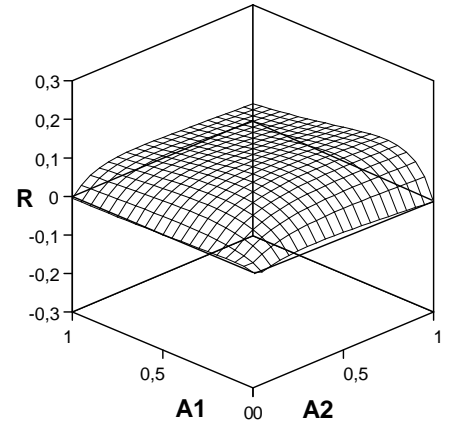

ascorbic acid-trolox

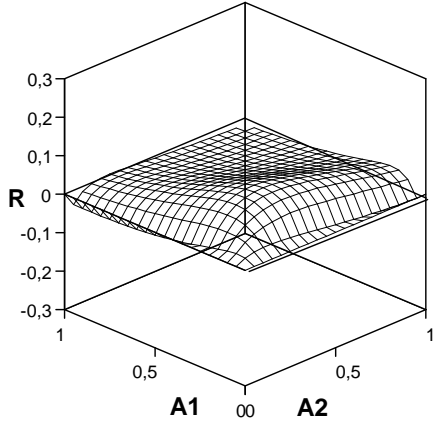

tocopherol-BHA

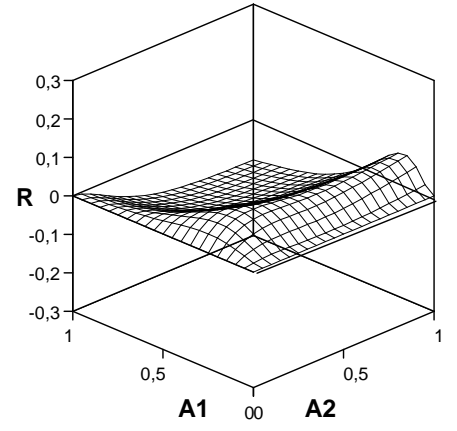

hypothetical

Figure 5: Differences between best-fit and null interaction responses in the specified cases. Hypothetical example was obtained by assuming independent action, with the following parametric values: $K_{1}=K_{2}=0.7 ; m_{1}=m_{2}=0.25 ; a_{1}=a_{2}=1.5 ; c_{2 \mathrm{~m}}=2 ; c_{1 \mathrm{k}}=1$. 


\section{TABLES}

Table 1: Joint action of TOC $\left(\mathrm{A}_{1}\right)$ and BHA $\left(\mathrm{A}_{2}\right)$ on $\beta$-carotene oxidation. The null interaction and synergy hypotheses are compared under the independent action and addition concentration suppositions, by fitting the experimental results to the (10) and (15) generalized models. $\theta$. parametric estimations; $C I \%$ : confidence intervals $(\alpha=0.05)$ as $\%$ of the parametric estimations; $R^{2}$ : adjusted coefficient of multiple determination. See Figure 3 and text for details.

\begin{tabular}{|c|c|c|c|c|c|c|c|}
\hline \multicolumn{4}{|c|}{ independent action } & \multicolumn{4}{|c|}{ concentration addition } \\
\hline & & $\begin{array}{c}\text { null } \\
\text { interaction }\end{array}$ & synergy & \multicolumn{3}{|r|}{$\begin{array}{c}\text { null } \\
\text { interaction }\end{array}$} & synergy \\
\hline \multirow{3}{*}{ response to $A_{1}$} & $\mathrm{~K}_{1}$ & $0.576 \pm 29.5$ & $0.566 \pm 7.2$ & \multirow{3}{*}{ joint response } & $\mathrm{K}$ & $0.785 \pm 6.1$ & $0.761 \pm 1.6$ \\
\hline & $\mathrm{m}_{1}$ & \multirow{2}{*}{$\begin{array}{l}0.388 \pm 38.0 \\
1.403+44.9\end{array}$} & $0.362 \pm 18.7$ & & $\mathrm{~m}$ & $0.263 \pm 16.0$ & $0.326 \pm 8.4$ \\
\hline & $\mathrm{a}_{1}$ & & $1.237 \pm 21.0$ & & $\mathrm{a}$ & $1.033 \pm 15.0$ & $0.895 \pm 6.6$ \\
\hline \multirow{3}{*}{ response to $\mathrm{A}_{2}$} & $\mathrm{~K}_{2}$ & $0.677 \pm 22.2$ & $0.589 \pm 10.7$ & relative potency & $\mathrm{p}$ & $0.667 \pm 17.9$ & $0.609 \pm 11.4$ \\
\hline & $\mathrm{m}_{2}$ & \multirow{2}{*}{$\begin{array}{l}0.256 \pm 38.4 \\
0.958 \pm 36.2\end{array}$} & $0.259 \pm 16.5$ & \multirow{2}{*}{$\begin{array}{l}\mathrm{A}_{1} \text { altering eff. } \\
\text { conc. of } \mathrm{A}_{2}\end{array}$} & $\mathrm{~b}_{2 \mathrm{D}}$ & $-\quad \quad-$ & $-\quad-$ \\
\hline & $\mathrm{a}_{2}$ & & $1.244 \pm 23.2$ & & $\mathrm{c}_{2 \mathrm{D}}$ & - & - \\
\hline \multirow{4}{*}{$\begin{array}{l}A_{1} \text { as perturbing } \\
\text { factor for params. } \\
\text { of the response to } \\
A_{2}\end{array}$} & $\mathrm{~b}_{2 \mathrm{k}}$ & $-\quad-$ & $-\quad \quad-$ & \multirow{2}{*}{$\begin{array}{l}\mathrm{A}_{2} \text { altering } \\
\text { eff. conc. of } \mathrm{A}_{1}\end{array}$} & $\mathrm{~b}_{1 \mathrm{D}}$ & - & $12.24 \pm 22.8$ \\
\hline & $\mathrm{c}_{2 \mathrm{k}}$ & - & - & & $\mathrm{c}_{1 \mathrm{D}}$ & - & $-\quad \quad-$ \\
\hline & $b_{2 m}$ & - & - & \multirow{4}{*}{$\begin{array}{l}\mathrm{A}_{1} \text { as perturbing } \\
\text { factor for params. } \\
\text { of the joint } \\
\text { response }\end{array}$} & $\mathrm{b}_{2 \mathrm{k}}$ & - & - \\
\hline & $\mathrm{c}_{2 \mathrm{~m}}$ & - & - & & $c_{2 k}$ & - & - \\
\hline \multirow{4}{*}{$\begin{array}{l}\mathrm{A}_{2} \text { as perturbing } \\
\text { factor for params. } \\
\text { of the response to } \\
\mathrm{A}_{1}\end{array}$} & $\mathrm{~b}_{1 \mathrm{k}}$ & - & - & & $\mathrm{b}_{2 \mathrm{~m}}$ & - & - \\
\hline & $\mathrm{c}_{1 \mathrm{k}}$ & - & - & & $c_{2 m}$ & - & - \\
\hline & $\mathrm{b}_{1 \mathrm{~m}}$ & - & $-\quad \quad-$ & \multirow{4}{*}{$\begin{array}{l}A_{2} \text { as perturbing } \\
\text { factor for params. } \\
\text { of the joint } \\
\text { response }\end{array}$} & $\mathrm{b}_{1 \mathrm{k}}$ & - & - \\
\hline & $\mathrm{c}_{1 \mathrm{~m}}$ & - & $4.006 \pm 44.5$ & & $\mathrm{c}_{1 \mathrm{k}}$ & - & - \\
\hline \multirow[t]{3}{*}{ comp / coop } & $\mathrm{s}$ & - & $-\quad \quad-$ & & $\mathrm{b}_{1 \mathrm{~m}}$ & - & - \\
\hline & & & & & $\mathrm{c}_{1 \mathrm{~m}}$ & - & - \\
\hline & $\mathbf{R}_{\text {adj }}^{2}$ & 0.9139 & 0.9693 & & $\mathbf{R}_{\text {adj }}^{2}$ & 0.9411 & 0.9907 \\
\hline
\end{tabular}


Table 2: Parametric estimations for the joint action of the following particular cases. $A_{1}$ and $A_{2}$ are the first and second element, respectively, of each pair. Other notations as in Table 1. See Figure 4 and text for details.

\begin{tabular}{|c|c|c|c|c|c|}
\hline $\begin{array}{l}\mathbf{A}_{1}: \\
\mathbf{A}_{2}:\end{array}$ & & $\begin{array}{c}\text { AA } \\
\text { ETX }\end{array}$ & $\begin{array}{l}\text { AA } \\
\text { Mn }\end{array}$ & $\begin{array}{c}\text { AA } \\
\text { TRO }\end{array}$ & $\begin{array}{l}\text { BHT } \\
\text { TRO }\end{array}$ \\
\hline \multirow{3}{*}{ response to $A_{1}$} & $\mathrm{~K}_{1}$ & $0.700 \pm 39.1$ & $0.568 \pm 13.2$ & $0.668 \pm 16.2$ & $0.597 \pm 22.1$ \\
\hline & $\mathrm{m}_{1}$ & $0.139 \pm 21.5$ & $0.100 \pm 21.2$ & $0.105 \pm 16.4$ & $0.881 \pm 28.4$ \\
\hline & $\mathrm{a}_{1}$ & $0868 \pm 14.9$ & $0.899 \pm 23.4$ & $0.884 \pm 15.1$ & $0.956 \pm 16.6$ \\
\hline \multirow{3}{*}{ response to $A_{2}$} & $\mathrm{~K}_{2}$ & $0.657 \pm 22.2$ & $0.614 \pm 5.2$ & $0.830 \pm 17.5$ & $0.660 \pm 21.3$ \\
\hline & $\mathrm{m}_{2}$ & $0.100 \pm 19.2$ & $0.305 \pm 14.1$ & $0.153 \pm 16.2$ & $0.117 \pm 21.6$ \\
\hline & $\mathrm{a}_{2}$ & $0.759 \pm 32.1$ & $1.005 \pm 23.2$ & $0.668 \pm 16.6$ & $1.068 \pm 18.3$ \\
\hline \multirow{4}{*}{$\begin{array}{l}A_{1} \text { as perturbing factor for } \\
\text { params. of the response to } \\
A_{2}\end{array}$} & $\mathrm{~b}_{2 \mathrm{k}}$ & - & -0.836 & - & - \\
\hline & $\mathrm{c}_{2 \mathrm{k}}$ & - & -0.759 & - & $0.314 \pm 8.8$ \\
\hline & $b_{2 m}$ & - & - & - & - \\
\hline & $\mathrm{c}_{2 \mathrm{~m}}$ & - & - & - & - \\
\hline \multirow{4}{*}{$\begin{array}{l}A_{2} \text { as perturbing factor for } \\
\text { params. of the response to } \\
A_{1}\end{array}$} & $\mathrm{~b}_{1 \mathrm{k}}$ & - & - & - & - \\
\hline & $\mathrm{c}_{1 \mathrm{k}}$ & - & - & - & - \\
\hline & $\mathrm{b}_{1 \mathrm{~m}}$ & - & - & - & - \\
\hline & $\mathrm{c}_{1 \mathrm{~m}}$ & $2.115 \pm 11.1$ & - & $2.621 \pm 6.6$ & - \\
\hline \multirow[t]{2}{*}{ comp / coop } & $\mathrm{s}$ & - & - & $0.980 \pm 1.6$ & - \\
\hline & $\mathbf{R}_{\text {adj }}^{2}$ & 0.9807 & 0.9876 & 0.9932 & 0.9942 \\
\hline
\end{tabular}


Table 3: Effect of the combination of 42 different pairs of antioxidants for each reaction. In the cases one antioxidant is combined with itself is used simply as a control. For each case the $R U V(\%)$ is presented. Note, that the underline combinations are those that have been analyzed in detail in the text.

A: $\beta$-CAROTENE REACTION (LIPOPHILIC)

\begin{tabular}{llcccccc}
\hline & \multicolumn{2}{c}{ BHA } & TRO & TOC & ETO & PG & BHT \\
\hline$(0-5 \mu \mathrm{M})$ & BHA & $N I-C A(0.0 \%)$ & $S-C A(12.2 \%)$ & $\underline{S-C A}(3.5 \%)$ & $S-I A(2.4 \%)$ & $A-I A(-9.6 \%)$ & $S-I A(1.6 \%)$ \\
$(0-300 \mu \mathrm{M})$ & TRO & - & $N I-C A(0.0 \%)$ & $A-I A(-0.5 \%)$ & $A-I A(-3.7 \%)$ & $A-C A(-2.1 \%)$ & $A-I A(-11.7 \%)$ \\
$(0-1 \mu \mathrm{M})$ & TOC & - & - & $N I-C A(0.0 \%)$ & $S-I A(5.3 \%)$ & $S-C A(1.3 \%)$ & $S-I A(1.9 \%)$ \\
$(0-2 \mathrm{nM})$ & ETO & - & - & - & $N I-C A(0.0 \%)$ & $A-C A(-4.8 \%)$ & $S-I A(20.3 \%)$ \\
$(0-80 \mu \mathrm{M})$ & PG & - & - & - & - & $N I-C A(0.0 \%)$ & $S-I A(0.3 \%)$ \\
$(0-30 \mu \mathrm{M})$ & BHT & - & - & - & - & $N I-C A(0.0 \%)$ \\
\hline
\end{tabular}

\section{B: CROCIN REACTION (HYDROPHILIC)}

\begin{tabular}{llcccccc}
\hline & & BHA & TRO & ETO & Mn & PG & AA \\
\hline$(0-350 \mu \mathrm{M})$ & BHA & $N I-C A(0.0 \%)$ & $A-C A(-2.9 \%)$ & $A-C A(-3.5 \%)$ & $S-I A(6.3 \%)$ & $S-C A(2.2 \%)$ & $S-I A(0.7 \%)$ \\
$(0-150 \mu \mathrm{M})$ & TRO & - & $N I-C A(0.0 \%)$ & $A-I A(-6.1 \%)$ & $S-C A(5.8 \%)$ & $S-I A(4.3 \%)$ & $\underline{S-I A}(7.7 \%)$ \\
$(0-60 \mu \mathrm{M})$ & ETO & - & - & $N I-C A(0.0 \%)$ & $S-I A(7.3 \%)$ & $S-I A(4.5 \%)$ & $\underline{S-I A}(9.4 \%)$ \\
$(0-10 \mu \mathrm{M})$ & Mn & - & - & - & $N I-C A(0.0 \%)$ & $S-C A(4.7 \%)$ & $\underline{A-I A}(-4.2 \%)$ \\
$(0-300 \mu \mathrm{M})$ & PG & - & - & - & $N I-C A(0.0 \%)$ & $S-C A(1.8 \%)$ \\
$(0-400 \mu \mathrm{M})$ & AA & - & - & - & - & - & $N I-C A(0.0 \%)$ \\
\hline
\end{tabular}

NI: Null interaction / S: Synergy / A: Antagonism / IA: Independent action / CA: Concentration addition 


\section{APPENDIX SECTION}

\section{Model selection criteria.}

In order to assist us select the best model, we have used different model selection criteria (MSC) to evaluate the multivariable fit and explanatory appropriateness of the equations. In the present work, the AIC, AICc, BIC, RIC, Cp, ${ }^{2}$ adj, FPE, and MSlC criteria (Table A1) were obtained directly using an Excel spreadsheet. The usefulness of MSC to choose the best solution and model is well-documented (Rivers \& Vuong, 2002). A model should be complex enough to extract the regularities in data, but simple enough not to overfit it and thereby reduce predictiveness. MSC adjust the goodness of fit in order to penalize model complexity, overfitting and lack of generalizability. Currently, there are a variety of MSC available (Forster, 2000; Myung \& Pitt, 2004), but there is no one criterion that can lead to a perfect choice (Roland T. Rust, Simester, Brodie, \& Nilikant, 1995).

If the above solutions do no solve completely the selection, other criteria more intuitively can be used, such as the asymmetric, kurtosis and distribution of the residuals. The residuals should be randomly scattered around zero to avoid autocorrelation (Roland T. Rust et al., 1995). These residuals should not be grouped and should not increase or decrease as a function of the independent variables. Hereafter, we will call OP the point's distribution that correlates, with a coefficient $r^{2}$, observed and predicted results, and $R^{2}$ the adjusted coefficient of multiple determination.

Model selection criteria help to differentiate the most "true solution". In general, all statistical MSC merge into similar solutions. Such a conclusion, can be explained, because once the solutions that do not present significant parameters are excluded, any of the MSC presented will solve similar and precisely the selection most appropriate.

In Table A2, an illustrative summary of the application of the different MSC used to evaluate the results obtained for the case study of BHA and tocopherol presented in the manuscript is shown. 
Table A1: Comparison of different model selection criteria (MSC) typically used to compare the models based in their complexity, goodness of fit, overfitting providing criteria to choose the most "true" solution. n: number of independent measurements considered in the fit. k: number of fitted parameters. RSS: residual sum of squares. ESS: explained sum of squares.

\begin{tabular}{|c|c|c|c|c|c|}
\hline Criterion & Ranking & Claim & Formula & Additional information & References \\
\hline $\begin{array}{l}\text { Akaike Information } \\
\text { Criterion (AIC) }\end{array}$ & Smaller value & $\begin{array}{l}\text { complexity } \\
\text { (efficient) }\end{array}$ & $A I C=n \ln \left(\frac{R S S}{n}\right)+2 k$ & It favors models with many variables. & $\begin{array}{l}\text { (Gang \& George, 1988; Shi } \\
\text { \& Tsai, 2002) }\end{array}$ \\
\hline $\begin{array}{l}\text { Akaike Information } \\
\text { Criterion Corrected (AICc) }\end{array}$ & Smaller value & $\begin{array}{l}\text { complexity } \\
\text { (efficient) }\end{array}$ & $A I C_{C}=n \ln \left(\frac{R S S}{n}\right)+\left(\frac{2(k+1)}{n-k-2}\right)$ & $\begin{array}{l}\text { It favors models with many variables, but } \\
\text { penalizes the complexity of the models in } \\
\text { larger way than the AIC. }\end{array}$ & $\begin{array}{l}\text { (Gang \& George, 1988; Shi } \\
\text { \& Tsai, 2002) }\end{array}$ \\
\hline $\begin{array}{l}\text { The Schwartz or Bayesan } \\
\text { Information Criterion (BIC } \\
\text { or SIC) }\end{array}$ & Smaller value & $\begin{array}{l}\text { complexity } \\
\text { (consistent) }\end{array}$ & $B I C=n \ln (R S S)+\ln (n) k$ & $\begin{array}{l}\text { The BIC is Bayesian because it is } \\
\text { designed as an index of the evidence in } \\
\text { favor of a given model being "true". }\end{array}$ & (Schwarz, 1978) \\
\hline $\begin{array}{l}\text { Akaike's Final Prediction } \\
\text { Error (FPE) }\end{array}$ & Smaller value & goodness of fit & $F P E=n \frac{R S S(n+k)}{(n-k)}$ & & (Shi \& Tsai, 2002) \\
\hline Mallows' criteria $(\mathrm{Cp})$ & Smaller value & $\begin{array}{l}\text { goodness of fit / } \\
\text { overfitting }\end{array}$ & $C_{P}=n[R S S /(E S S / n-1)]-n+2 k$ & & $\begin{array}{l}\text { (Gang \& George, 1988; Shi } \\
\& \text { Tsai, 2002) }\end{array}$ \\
\hline $\begin{array}{l}\text { Adjusted Coefficient of } \\
\text { determination } \\
\left(R^{2}{ }_{\text {adj }}\right)\end{array}$ & Highest value & $\begin{array}{l}\text { goodness of fit / } \\
\text { complexity }\end{array}$ & $R_{a d j}^{2}=\frac{(n-1) R^{2}-k}{n-1-k}$ & $\begin{array}{l}\text { The proposed adjusted coefficients correct } \\
\text { the overestimation problem of the } \\
\text { unadjusted coefficients. }\end{array}$ & (Shi \& Tsai, 2002) \\
\hline $\begin{array}{l}\text { Residual Information } \\
\text { Criterion (RIC) }\end{array}$ & Smaller value & $\begin{array}{l}\text { goodness of fit / } \\
\text { overfitting }\end{array}$ & $R I C=(n-k) \ln (R S S)+k[\ln (n)-1]+\frac{4}{n-k-2}$ & $\begin{array}{l}\text { Performs well except when the sample } \\
\text { size is small and the signal-to-noise ratio } \\
\text { is weak. RIC's large penalty function } \\
\text { allows it to perform better than BIC. }\end{array}$ & (Shi \& Tsai, 2002) \\
\hline $\begin{array}{l}\text { Model Selection Criterion } \\
(\mathrm{MSIC})\end{array}$ & Highest value & goodness of fit & $M S C=\ln \left(\frac{E S S}{R S S}\right)-\frac{2 k}{n}$ & & (Schwarz, 1978) \\
\hline
\end{tabular}


Table A2: Model ranking (Rk) obtained for each MSC for the TOC and BHA case ( $\beta$-carotene bleaching reaction). Two different rankings are shown, one taking into account the results of both modes of action, and another (in brackets) that only considers the results for each hypothesis. For each mode of action the $\mathrm{C}-1$ is the statistical results found for the null interaction, and the other four cases are the top cases that best fit the joint action of TOC and BHA.

\begin{tabular}{|c|c|c|c|c|c|c|c|c|c|c|c|c|c|c|c|c|c|c|c|c|c|c|}
\hline \multirow{3}{*}{\multicolumn{2}{|c|}{ CASES }} & \multicolumn{5}{|c|}{ STATISTICS } & \multicolumn{16}{|c|}{ MODEL SELECTION CRITERIA } \\
\hline & & \multirow{2}{*}{$\mathbf{k}$} & \multirow{2}{*}{ RSS } & \multirow{2}{*}{$\mathbf{R}_{\text {adj }}^{2}$} & \multirow{2}{*}{ ESS } & \multirow{2}{*}{$\mathbf{S}^{2}$} & \multicolumn{2}{|c|}{ AIC } & \multicolumn{2}{|c|}{ AICc } & \multicolumn{2}{|c|}{ BIC } & \multicolumn{2}{|c|}{ FPE } & \multicolumn{2}{|c|}{$\mathbf{R}_{\text {adj }}^{2}$} & \multicolumn{2}{|c|}{ RIC } & \multicolumn{2}{|c|}{ Cp } & \multicolumn{2}{|c|}{ MSC } \\
\hline & & & & & & & Value & Rk & Value & Rk & Value & Rk & Value & Rk & Value & Rk & Value & $\mathbf{R k}$ & Value & Rk & Value & Rk \\
\hline \multirow{5}{*}{ IA } & C-1 & 6 & 0.310 & 0.9139 & 3.20 & 0.0409 & -353.9 & $10(5)$ & -365.7 & $10(5)$ & -74.8 & $10(5)$ & 16.25 & 10(5) & 0.9455 & $9(5)$ & -71.4 & 10(5) & 261.7 & $10(5)$ & 2.37 & $10(5)$ \\
\hline & $\mathrm{C}-2$ & 6 & 0.120 & 0.9627 & 2.80 & 0.0445 & -389.9 & $8(4)$ & -401.6 & $8(4)$ & -110.8 & $7(3)$ & 9.27 & $8(4)$ & 0.9588 & $8(4)$ & -104.0 & $7(3)$ & 120.6 & $8(4)$ & 2.96 & $7(3)$ \\
\hline & $\mathrm{C}-3$ & 7 & 0.085 & 0.9628 & 3.14 & 0.0499 & -409.3 & $6(2)$ & -423.0 & $6(2)$ & -128.0 & $6(2)$ & 6.84 & $6(2)$ & 0.9694 & $6(2)$ & -117.8 & $6(2)$ & 60.2 & $6(2)$ & 3.38 & $6(2)$ \\
\hline & C-4 & 8 & 0.110 & 0.9667 & 2.73 & 0.0435 & -391.3 & 7(3) & -406.9 & 7(3) & -107.8 & $8(4)$ & 9.08 & $7(3)$ & 0.9618 & $7(3)$ & -98.1 & $8(4)$ & 114.4 & $7(3)$ & 2.96 & $8(4)$ \\
\hline & C-5 & 8 & 0.080 & 0.9693 & 2.76 & 0.0486 & -474.0 & 4(1) & -489.6 & $4(1)$ & -190.5 & $5(1)$ & 2.49 & $4(1)$ & 0.9888 & $4(1)$ & -170.5 & $5(1)$ & -8.1 & $5(1)$ & 4.37 & $4(1)$ \\
\hline \multirow{5}{*}{$\mathrm{CA}$} & C-1 & 4 & 0.165 & 0.9411 & 2.91 & 0.0463 & -373.3 & $9(5)$ & -381.1 & $9(5)$ & -98.5 & $9(5)$ & 12.00 & $9(5)$ & 0.9431 & $10(5)$ & -95.2 & $9(5)$ & 172.6 & $9(5)$ & 2.75 & $9(5)$ \\
\hline & C-2 & 6 & 0.029 & 0.9906 & 3.02 & 0.0481 & -479.6 & 2(2) & -491.3 & 2(2) & -200.5 & 2(2) & 2.28 & $2(2)$ & 0.9896 & 2(2) & -185.3 & 2(2) & -12.7 & $3(3)$ & 4.44 & $3(3)$ \\
\hline & C-3 & 6 & 0.029 & 0.9905 & 3.05 & 0.0485 & -479.2 & $3(3)$ & -491.0 & $3(3)$ & -200.1 & $3(3)$ & 2.29 & $3(3)$ & 0.9895 & 3(3) & -184.9 & $3(3)$ & -12.8 & 2(2) & 4.45 & 2(2) \\
\hline & C-4 & 4 & 0.028 & 0.9907 & 3.04 & 0.0482 & -481.2 & 1(1) & -491.4 & 1(1) & -204.3 & 1(1) & 2.22 & $1(1)$ & 0.9899 & 1(1) & -191.6 & $1(1)$ & -14.6 & $1(1)$ & 4.47 & $1(1)$ \\
\hline & C-5 & 5 & 0.033 & 0.9892 & 3.03 & 0.0482 & -473.4 & $5(4)$ & -483.2 & $5(4)$ & -196.5 & 4(4) & 2.51 & $5(4)$ & 0.9883 & 5(4) & -184.4 & $4(4)$ & -9.4 & $4(4)$ & 4.35 & $5(4)$ \\
\hline
\end{tabular}

k: number of fitted parameters; RSS: residual sum of squares; ESS: explained sum of squares; $S^{2}$ : standard deviation 


\section{Experimental design}

In any design, a convenient practice is to code the doses (dividing them by the maximum ones) in such a way that both individual series include the same values $\left(D_{\mathrm{i}}\right)$ within the $[0,1]$ interval. Together with the encoding of the response in the same interval, this facilitates the fitting process and provides standardized parametric estimates. Once the $D_{\mathrm{i}}$ series is defined, there are several reasonable modes to establish the mixed doses covering the experimental domain (Figure A1).

\section{Simple radial design}

Besides the individual series $D_{1 \mathrm{i}}, 0$ and $D_{2 \mathrm{i}}, 0\left(D_{1 \mathrm{i}}=D_{2 \mathrm{i}}=D_{\mathrm{i}}\right)$, this option includes several additional sets of mixed doses $\left(d_{1 \mathrm{i}}, d_{2 \mathrm{i}}\right)$, each set defined by a constant ratio $\left(d_{1 \mathrm{i}} / d_{2 \mathrm{i}}=Q\right)$ between the concentrations of both effectors. Thus, the mixed dose set located along the radius defined by $Q_{\mathrm{n}}$ is:

$$
\begin{aligned}
& \text { If } Q_{\mathrm{n}} \leq 1: d_{1 i\left[Q_{n}\right]}=D_{i} \quad ; \quad d_{2 i\left[Q_{n}\right]}=D_{i} \times Q_{n} \\
& \text { If } Q_{\mathrm{n}}>1: d_{1 i\left[Q_{n}\right]}=D_{i} / Q_{n} \quad ; \quad d_{2 i\left[Q_{n}\right]}=D_{i}
\end{aligned}
$$

\section{Concentric radial design}

Similar to the preceding one, but with mixed doses defined from the angle $\left(\varphi_{n}\right)$ that each radius makes with the variable representing the $D_{1 \mathrm{i}}$ series:

$$
d_{1 \mathrm{i}\left[\varphi_{\mathrm{n}}\right]}=D_{i} \times \cos \varphi_{\mathrm{n}} \quad ; \quad d_{2 \mathrm{i}\left[\varphi_{\mathrm{n}}\right]}=D_{i} \times \sin \varphi_{\mathrm{n}}
$$

Number of radii and values of $\varphi_{\mathrm{j}}$ (or $Q$ ) can be freely fixed, taking into account that high $\left(\sim 75^{\circ}\right)$ and low $\left(\sim 15^{\circ}\right)$ values of $\varphi_{\mathrm{j}}$ favor the detection of interactions.

\section{Equiadditive design}

Mixed doses are grouped in series defined by a constant sum $\left(d_{1 \mathrm{i}}+d_{2 \mathrm{i}}=S\right)$. Thus, $v$ being the desired number of doses per series:

$$
\begin{aligned}
& \text { If } S_{\mathrm{n}} \leq 1: d_{1 i\left[S_{n}\right]}=S_{n}-h_{v}\left(\frac{S_{n}}{v-1}\right) \quad ; \quad d_{2 i\left[S_{n}\right]}=S_{n}-d_{1 i\left[S_{n}\right]} ; \quad\left(h_{\mathrm{v}}=0,1, \ldots v-1\right) \\
& \text { If } S_{\mathrm{n}}>1: d_{1 i\left[S_{n}\right]}=\left(S_{n}-1\right)+h_{v}\left(\frac{1-S_{n}+1}{v-1}\right) ; d_{2 i\left[S_{n}\right]}=S_{n}-d_{1 i\left[S_{n}\right]} ; \quad\left(h_{\mathrm{v}}=0,1, \ldots v-1\right)
\end{aligned}
$$

Radial equiadditive design: mixed doses fulfill simultaneously the conditions $d_{1 \mathrm{i}} / d_{2 \mathrm{i}}=Q_{\mathrm{n}}$ and $d_{1 \mathrm{i}}+d_{2 \mathrm{i}}=S_{\mathrm{n}}$, therefore:

$$
d_{1 i}=S_{n} /\left(1+Q_{n}\right) \quad ; \quad d_{2 i}=S_{n}\left[1-\left(1 /\left(1+Q_{n}\right)\right)\right]
$$

\section{Complete design}

It is the most intuitive experimental plan, combining simply all the doses of an effector with all doses of the other.

In principle, each design offers specific advantages for identifying concrete modes of action and interaction by comparing, through an appropriate statistical criterion, the observed responses at certain dose series with the expected ones under IA or CA null interaction hypotheses. However, in our 
experience the response surface properties in joint actions imply: 1) numerous indistinguishable situations as analyzed by means of radial or equiadditive series; 2) responses whose behavior in a given region of the experimental domain does not represent necessarily what takes place in other regions.

In fact, the most discriminative tool is the explicit model, and in order to simulate such a conditions, the complete design is the most advisable. Even if one wants to disregard doubtful auxiliary functions, the responses to a same dose set of an effector in the presence of increasing doses of the another form very specific systematic sequences. These sequences are more informative than radial or equiadditive ones, and can be advantageously subjected to the comparative criteria above mentioned. Additionally, a good coverage of the experimental domain (complete design) is more efficient than an increase of the number of replicates to minimize the effects of the experimental error.
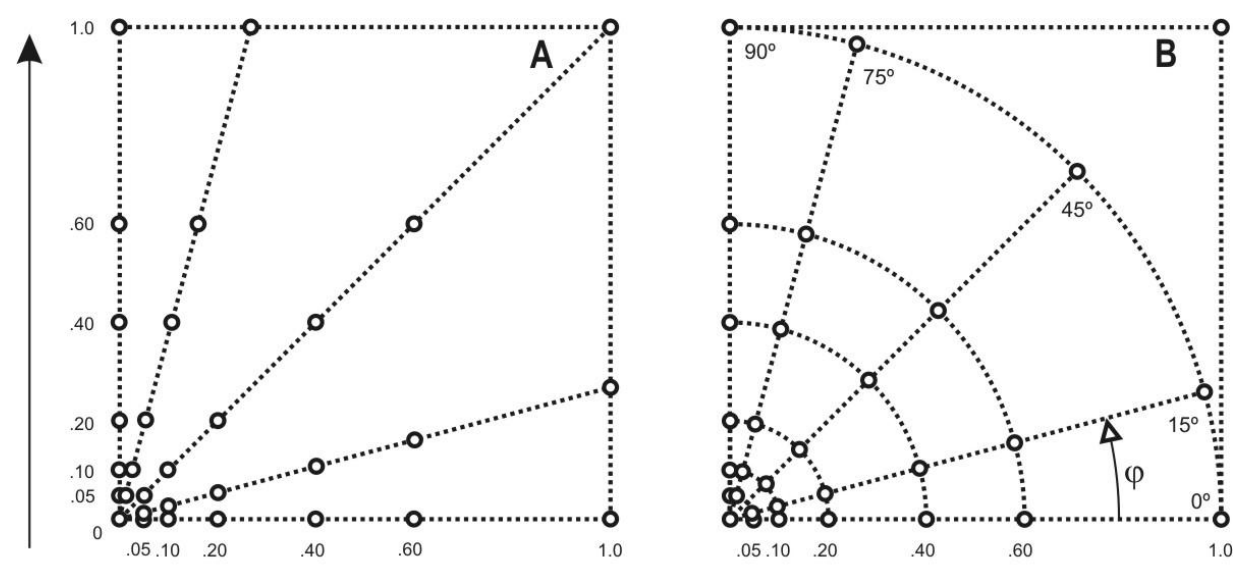

D2
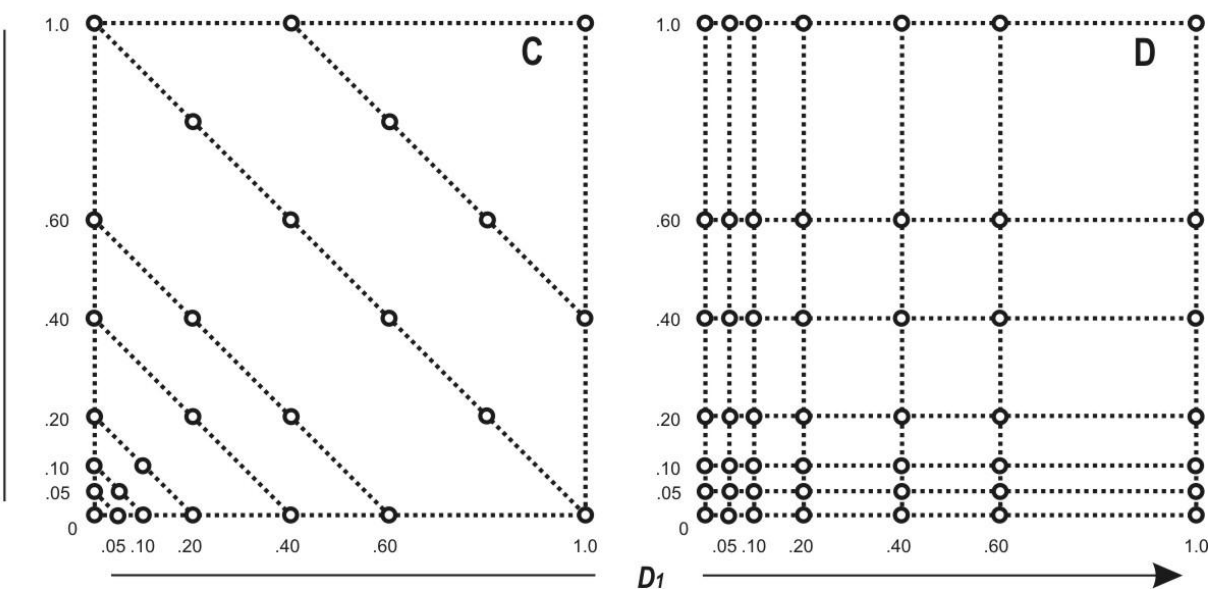

Figure A1: simple radial (A), concentric radial (B), equiadditive (C) and complete (D) designs. 


\section{Full antioxidant pairs combination analysis}

\subsubsection{Crocin bleaching reaction}

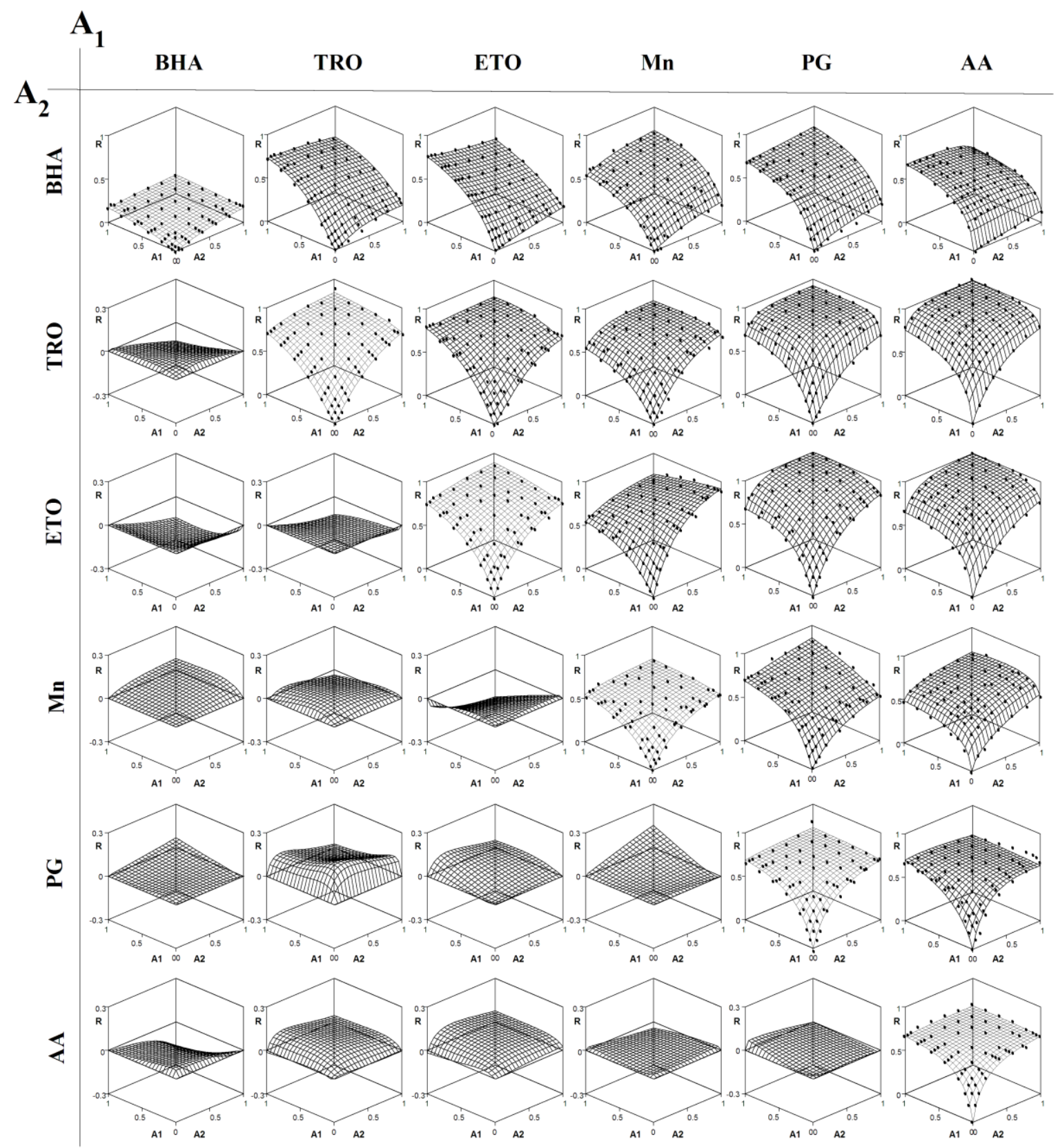

Figure A2: Matrix combination responses for the crocin bleaching reaction, which is organized as follows: a) in the diagonal it can be seem the results obtained for the controls; b) in the top part of the diagonal the surface responses for each pair antioxidant combination is presented; and c) in the bottom diagonal part the differences "scenery" between their respective null interaction form and the obtained response is presented. Numerical results in Table A3. 
Table A3: Parametric values of the joint action of six different antioxidants in the crocin oxidation reaction. The null interaction and synergy hypotheses are compared under the independent action and addition concentration suppositions, by fitting the experimental results to the (10) and (15) generalized models. See Figure A2 and text for details. In all the presented results the parameters estimations are significant.

\section{INDEPENDENT ACTION (IA)}

\begin{tabular}{|c|c|c|c|c|c|c|c|c|c|c|}
\hline $\begin{array}{l}\mathbf{A}_{1} \\
\mathbf{A}_{2}\end{array}$ & & $\begin{array}{l}E T X \\
T R O\end{array}$ & $\begin{array}{c}M n \\
B H A\end{array}$ & $\begin{array}{c}M n \\
\text { ETX }\end{array}$ & $\begin{array}{c}P G \\
T R O\end{array}$ & $\begin{array}{c}P G \\
E T X\end{array}$ & $\begin{array}{c}A A \\
B H A\end{array}$ & $\begin{array}{c}A A \\
T R O\end{array}$ & $\begin{array}{c}A A \\
E T X\end{array}$ & $\begin{array}{l}A A \\
M n\end{array}$ \\
\hline \multirow{3}{*}{ response to $A_{l}$} & $K_{1}$ & 0.904 & 0.606 & 0.614 & 0.722 & 0.701 & 0.730 & 0.668 & 0.700 & 0.568 \\
\hline & $m_{1}$ & 0.369 & 0.302 & 0.305 & 0.197 & 0.187 & 0.149 & 0.105 & 0.139 & 0.100 \\
\hline & $a_{1}$ & 1.041 & 0.985 & 1.005 & 0.905 & 0.959 & 0.669 & 0.884 & 0868 & 0.899 \\
\hline \multirow{3}{*}{ response to $A_{2}$} & $K_{2}$ & 0.710 & 0.308 & 0.997 & 0.804 & 0.992 & 0.302 & 0.830 & 0.657 & 0.614 \\
\hline & $m_{2}$ & 0.380 & 0.457 & 0.248 & 0.386 & 0.319 & 0.657 & 0.153 & 0.100 & 0.305 \\
\hline & $a_{2}$ & 1.322 & 0.994 & 0.922 & 1.065 & 0.885 & 0.988 & 0.668 & 0.759 & 1.005 \\
\hline \multirow{4}{*}{$\begin{array}{r}A_{1} \text { as perturbing factor } \\
\text { for params. of the } \\
\text { response to } A_{2}\end{array}$} & $b_{2 k}$ & -0.903 & - & & - & - & 2.801 & - & - & -0.836 \\
\hline & $c_{2 k}$ & - & - & -0.771 & - & - & - & - & - & -0.759 \\
\hline & $b_{2 m}$ & - & - & - & - & - & - & - & - & - \\
\hline & $c_{2 m}$ & - & - & - & 2.804 & 2.338 & - & - & - & - \\
\hline \multirow{4}{*}{$\begin{array}{r}A_{2} \text { as perturbing factor } \\
\text { for params. of the } \\
\text { response to } A_{l}\end{array}$} & $b_{1 k}$ & - & - & - & - & - & - & - & - & - \\
\hline & $c_{l k}$ & - & - & - & - & 1.090 & - & - & - & - \\
\hline & $b_{1 m}$ & - & -0.305 & - & 1.682 & 5.358 & - & - & - & - \\
\hline & $c_{1 m}$ & - & - & - & 11.049 & - & - & 2.621 & 2.115 & - \\
\hline \multirow[t]{2}{*}{ comp / coop } & $s$ & 0.613 & - & - & 1.034 & 0.886 & - & 0.980 & - & - \\
\hline & $\mathbf{R}_{\text {adj }}^{2}$ & 0.9756 & 0.9991 & 0.9994 & 0.9997 & 0.9998 & 0.9798 & 0.9932 & 0.9807 & 0.9876 \\
\hline
\end{tabular}

\section{CONCENTRATION ADDITION (CA)}

\begin{tabular}{|c|c|c|c|c|c|c|c|c|c|c|c|c|c|}
\hline $\begin{array}{l}\mathbf{A}_{1} \\
\mathbf{A}_{2}\end{array}$ & & $\begin{array}{l}B H A \\
B H A\end{array}$ & $\begin{array}{l}T R O \\
T R O\end{array}$ & $\begin{array}{l}E T X \\
E T X\end{array}$ & $\begin{array}{l}M n \\
M n\end{array}$ & $\begin{array}{l}P G \\
P G\end{array}$ & $\begin{array}{l}A A \\
A A\end{array}$ & $\begin{array}{l}\text { TRO } \\
B H A\end{array}$ & $\begin{array}{l}E T X \\
B H A\end{array}$ & $\begin{array}{l}M n \\
T R O\end{array}$ & $\begin{array}{c}P G \\
B H A\end{array}$ & $\begin{array}{l}P G \\
M n\end{array}$ & $\begin{array}{l}A A \\
P G\end{array}$ \\
\hline \multirow{3}{*}{ joint response } & $K$ & 0.249 & 0.868 & 0.950 & 0.647 & 0.739 & 0.689 & 0.851 & 0.942 & 0.808 & 0.233 & 0.548 & 0.792 \\
\hline & $m$ & 0.599 & 0.437 & 0.372 & 0.336 & 0.208 & 0.129 & 1.720 & 4.181 & 0.344 & 0.393 & 0.292 & 0.270 \\
\hline & $a$ & 0.965 & 1.117 & 0.844 & 0.915 & 0.865 & 0.758 & 1.118 & 0.857 & 1.030 & 0.968 & 1.034 & 0.856 \\
\hline relative potency & $p$ & 1.035 & 1.001 & 1.000 & 1.000 & 1.006 & 1.000 & 4.616 & 11.372 & 0.961 & 5.808 & 2.161 & 1.596 \\
\hline \multirow{2}{*}{$\begin{array}{r}A_{1} \text { altering eff. conc. of } \\
A_{2}\end{array}$} & $b_{2 D}$ & - & - & - & - & - & - & - & - & 0.843 & - & -0.5571 & - \\
\hline & $c_{2 D}$ & - & - & - & - & - & - & - & - & -0.493 & - & - & - \\
\hline \multirow{2}{*}{$\begin{array}{r}A_{2} \text { altering } \\
\text { eff. conc. of } A_{I}\end{array}$} & $b_{1 D}$ & - & - & - & - & - & - & - & - & - & - & - & - \\
\hline & $c_{1 D}$ & - & - & - & - & - & - & - & 0.818 & - & 0.201 & - & - \\
\hline \multirow{4}{*}{$\begin{array}{r}A_{I} \text { as perturbing factor } \\
\text { for params. of the joint } \\
\text { response }\end{array}$} & $b_{2 k}$ & - & - & - & - & - & - & - & - & -0.051 & 8.321 & 0.266 & - \\
\hline & $c_{2 k}$ & - & - & - & - & - & - & - & - & - & 2.148 & - & 0.191 \\
\hline & $b_{2 m}$ & - & - & - & - & - & - & - & - & 0.599 & - & - & - \\
\hline & $c_{2 m}$ & - & - & - & - & - & - & - & - & - & - & - & - \\
\hline \multirow{5}{*}{$\begin{array}{r}A_{2} \text { as perturbing factor } \\
\text { for params. of the joint } \\
\text { response }\end{array}$} & $b_{1 k}$ & - & - & - & - & - & - & -0.194 & - & - & & 0.270 & - \\
\hline & $c_{l k}$ & - & - & - & - & - & - & - & - & - & -0.096 & - & - \\
\hline & $b_{1 m}$ & - & - & - & - & - & - & - & - & - & - & - & - \\
\hline & $c_{l m}$ & - & - & - & - & - & - & - & - & - & - & -0.452 & - \\
\hline & $\mathbf{R}_{\text {adj }}^{2}$ & 0.9985 & 0.9995 & 0.9998 & 0.9994 & 0.9985 & 0.9984 & 0.9937 & 0.9995 & 0.9998 & 0.9992 & 0.9989 & 0.9958 \\
\hline
\end{tabular}




\subsection{2. $\beta$-carotene bleaching reaction}

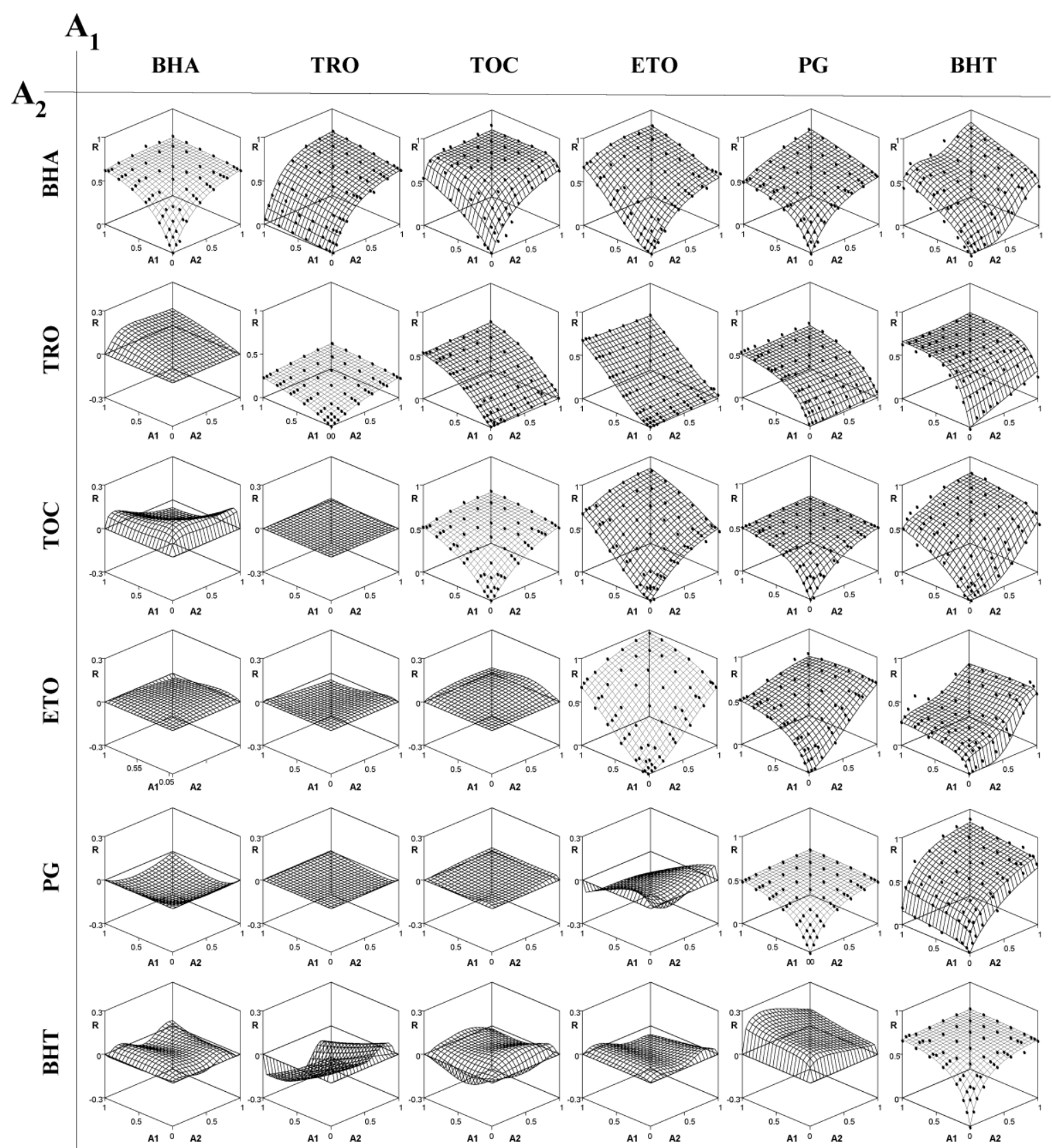

Figure A3: Matrix combination responses for the $\beta$-carotene bleaching reaction, which is organized as follows: a) in the diagonal it can be seem the results obtained for the controls; b) in the top part of the diagonal the surface responses for each pair antioxidant combination is presented; and c) in the bottom diagonal part the differences ("scenery") between their respective null interaction form and the obtained response is presented. Numerical results in Table A4. 
Table A4: Parametric values of the joint action of six different antioxidants in the $\beta$-carotene oxidation reaction. The null interaction and synergy hypotheses are compared under the independent action and addition concentration suppositions, by fitting the experimental results to the (10) and (15) generalized models. See Figure A3 and text for details. Note, that in all the presented results the parameters estimations are significant.

\section{INDEPENDENT ACTION (IA)}

\begin{tabular}{|c|c|c|c|c|c|c|c|c|c|c|c|}
\hline $\begin{array}{l}\mathbf{A}_{1} \\
\mathbf{A}_{2}\end{array}$ & & $\begin{array}{l}\text { TOC } \\
\text { TRO }\end{array}$ & $\begin{array}{l}E T X \\
B H A\end{array}$ & $\begin{array}{l}E T X \\
T R O\end{array}$ & $\begin{array}{l}E T X \\
T O C\end{array}$ & $\begin{array}{c}P G \\
B H A\end{array}$ & $\begin{array}{l}B H T \\
B H A\end{array}$ & $\begin{array}{l}\text { BHT } \\
T R O\end{array}$ & $\begin{array}{l}\text { BHT } \\
\text { TOC }\end{array}$ & $\begin{array}{l}\text { BHT } \\
E T X\end{array}$ & $\begin{array}{c}\text { BHT } \\
P G\end{array}$ \\
\hline \multirow{3}{*}{ response to $A_{l}$} & $\overline{K_{I}}$ & 0.583 & 1.000 & 1.000 & 1.000 & 0.514 & 1.000 & 0.597 & 0.597 & 0.154 & 0.374 \\
\hline & $m_{1}$ & 0.376 & 0.754 & 0.758 & 0.754 & 0.201 & 0.881 & 0.881 & 0.468 & 0.080 & 0.211 \\
\hline & $a_{1}$ & 1.184 & 1.629 & 1.567 & 1.584 & 0.960 & 0.837 & 0.956 & 1.236 & 1.090 & 0.395 \\
\hline \multirow{3}{*}{ response to $A_{2}$} & $\overline{K_{2}}$ & 0.410 & 0.594 & 0.100 & 0.576 & 0.591 & 0.528 & 0.660 & 1.000 & 0.861 & 0.621 \\
\hline & $m_{2}$ & 6.669 & 0.276 & 1.696 & 0.389 & 0.255 & 0.679 & 0.117 & 0.912 & 0.394 & 0.700 \\
\hline & $a_{2}$ & 0.926 & 1.039 & 0.781 & 1.135 & 0.973 & 4.031 & 1.068 & 2.522 & 0.655 & 4.456 \\
\hline \multirow{4}{*}{$\begin{array}{l}A_{1} \text { as perturbing factor for } \\
\text { params. of the response to } A_{2}\end{array}$} & $b_{2 k}$ & - & - & - & -0.447 & 0.498 & - & - & - & - & - \\
\hline & $c_{2 k}$ & - & - & - & - & - & -0.367 & 0.314 & - & - & - \\
\hline & $b_{2 m}$ & - & -0.235 & - & - & -0.747 & - & - & -0.475 & -0.669 & - \\
\hline & $c_{2 m}$ & -0.901 & - & 12.772 & -0.744 & - & - & - & - & - & -0.136 \\
\hline \multirow{4}{*}{$\begin{array}{l}A_{2} \text { as perturbing factor for } \\
\text { params. of the response to } A_{1}\end{array}$} & $b_{1 k}$ & 0.097 & -0.245 & - & - & -0.630 & -0.711 & - & - & - & - \\
\hline & $c_{l k}$ & - & - & - & - & - & - & - & - & - & - \\
\hline & $b_{1 m}$ & - & - & - & - & - & - & - & - & - & - \\
\hline & $c_{1 m}$ & - & - & - & 0.711 & - & 5.423 & - & - & - & 20.241 \\
\hline \multirow[t]{2}{*}{ comp / coop } & $s$ & - & - & -0.991 & 1.251 & - & - & - & - & - & 1.434 \\
\hline & $\mathbf{R}_{\text {adj }}^{2}$ & 0.9987 & 0.9997 & 0.9996 & 0.9988 & 0.9976 & 0.9923 & 0.9942 & 0.9892 & 0.9818 & 0.9785 \\
\hline
\end{tabular}

\section{CONCENTRATION ADDITION (CA)}

\begin{tabular}{|c|c|c|c|c|c|c|c|c|c|c|c|c|}
\hline $\begin{array}{l}\mathbf{A}_{1} \\
\mathbf{A}_{2}\end{array}$ & & $\begin{array}{l}B H A \\
B H A\end{array}$ & $\begin{array}{l}T R O \\
T R O\end{array}$ & $\begin{array}{l}\text { TOC } \\
\text { TOC }\end{array}$ & $\begin{array}{l}E T X \\
E T X\end{array}$ & $\begin{array}{l}P G \\
P G\end{array}$ & $\begin{array}{l}\text { BHT } \\
B H T\end{array}$ & $\begin{array}{l}\text { TRO } \\
B H A\end{array}$ & $\begin{array}{l}\text { TOC } \\
\text { BHA }\end{array}$ & $\begin{array}{l}P G \\
T R O\end{array}$ & $\begin{array}{l}P G \\
T O C\end{array}$ & $\begin{array}{l}P G \\
E T X\end{array}$ \\
\hline \multirow{3}{*}{ joint response } & $K$ & 0.674 & 0.995 & 0.599 & 1.000 & 0.508 & 0.694 & 0.843 & 0.761 & 0.572 & 0.624 & 1.000 \\
\hline & $m$ & 0.261 & 15.043 & 0.408 & 0.756 & 0.205 & 0.140 & 0.241 & 0.326 & 6.993 & 0.373 & 5.041 \\
\hline & $a$ & 0.978 & 0.894 & 1.166 & 1.558 & 0.971 & 0.806 & 1.199 & 0.895 & 1.067 & 1.151 & 0.869 \\
\hline relative potency & $p$ & 1.010 & 0.998 & 1.016 & 0.999 & 0.997 & 1.005 & 0.038 & 0.609 & 36.116 & 1.975 & 10.091 \\
\hline \multirow{2}{*}{$A_{1}$ altering eff. conc. of $A_{2}$} & $b_{2 D}$ & - & - & - & - & - & - & 0.531 & - & - & 1.682 & - \\
\hline & $c_{2 D}$ & - & - & - & - & - & - & - & - & 8.381 & 1.399 & -0.680 \\
\hline \multirow{2}{*}{$\begin{array}{r}A_{2} \text { altering } \\
\text { eff. conc. of } A_{1} \\
\end{array}$} & $b_{1 D}$ & - & - & - & - & - & - & - & 12.24 & - & - & - \\
\hline & $c_{1 D}$ & - & - & - & - & - & - & 13.320 & - & - & - & 7.262 \\
\hline \multirow{4}{*}{$\begin{array}{l}A_{l} \text { as perturbing factor for } \\
\text { params. of the joint response }\end{array}$} & $b_{2 k}$ & - & - & - & - & - & - & - & - & - & - & - \\
\hline & $c_{2 k}$ & - & - & - & - & - & - & - & - & - & - & 0.445 \\
\hline & $b_{2 m}$ & - & - & - & - & - & - & - & - & 0.912 & 1.588 & - \\
\hline & $c_{2 m}$ & - & - & - & - & - & - & - & - & - & - & - \\
\hline \multirow{5}{*}{$\begin{array}{l}A_{2} \text { as perturbing factor for } \\
\text { params. of the joint response }\end{array}$} & $b_{l k}$ & - & - & - & - & - & - & -0.038 & - & 0.098 & - & - \\
\hline & $c_{l k}$ & - & - & - & - & - & - & - & - & - & - & - \\
\hline & $b_{1 m}$ & - & - & - & - & - & - & 1.248 & - & 0.249 & - & - \\
\hline & $c_{l m}$ & - & - & - & - & - & - & - & - & - & -0.236 & 8.211 \\
\hline & $\mathbf{R}_{\text {adj }}^{2}$ & 0.9987 & 0.9981 & 0.9994 & 0.9996 & 0.9976 & 0.9981 & 0.9998 & 0.9881 & 0.9991 & 0.9987 & 0.9975 \\
\hline
\end{tabular}




\section{REFERENCES FROM THE APPENDIX SECTION}

Forster, M. R. (2000). Key Concepts in Model Selection: Performance and Generalizability. Journal of mathematical psychology, 44(1), 205-231.

Gang, Y., \& George, J. (1988). Statistical Model Selection Criteria. 28, 47-51.

Myung, J. I., \& Pitt, M. A. (2004). Model Comparison Methods. In Ludwig Brand and Michael L. Johnson, Methods in Enzymology(pp. 351-366). Academic Press.

Rivers, D., \& Vuong, Q. (2002). Model selection tests for nonlinear dynamic models. Econometrics Journal, 5(1), 1-39.

Roland T. Rust, Simester, D., Brodie, R. J., \& Nilikant, V. (1995). Model Selection Criteria: An Investigation of Relative Accuracy, Posterior Probabilities, and Combinations of Criteria. Management Science, 41(2), pp. 322-333.

Schwarz, G. (1978). Estimating the Dimension of a Model. The Annals of Statistics, 6(2), pp. 461-464.

Shi, P., \& Tsai, C. (2002). Regression Model Selection: A Residual Likelihood Approach. Journal of the Royal Statistical Society.Series B (Statistical Methodology), 64(2), pp. 237-252. 